\title{
Configuration space integral for long $n$-knots and the Alexander polynomial
}

\author{
TADAYUKI WATANABE
}

\begin{abstract}
There is a higher dimensional analogue of the perturbative Chern-Simons theory in the sense that a similar perturbative series as in 3 dimensions, which is computed via configuration space integral, yields an invariant of higher dimensional knots (BottCattaneo-Rossi invariant). This invariant was constructed by Bott for degree 2 and by Cattaneo-Rossi for higher degrees. However, its feature is yet unknown. In this paper we restrict the study to long ribbon $n$-knots and characterize the Bott-Cattaneo-Rossi invariant as a finite type invariant of long ribbon $n$-knots introduced by HabiroKanenobu-Shima [10]. As a consequence, we obtain a nontrivial description of the Bott-Cattaneo-Rossi invariant in terms of the Alexander polynomial.
\end{abstract}

57Q45; 57M25

\section{Introduction}

Witten [18] gave a path integral formulation of the Chern-Simons field theory which gives a framework for understanding many invariants of knots in a 3-manifold. But the path integral is not yet well defined mathematically. Though, the perturbative expansion method for the path integral gives a mathematical definition of knot invariants. It is defined by configuration space integral with certain uni-trivalent graphs called Jacobi diagrams. This construction of invariants has been studied by Axelrod-Singer [2], Bar-Natan [3], Guadagnini-Martellini-Mintchev [9], Kohno [12], Kontsevich [13], Bott-Taubes [5], Dylan Thurston [16] and others. It is known that the configuration space integral invariant of knots in $S^{3}$ is a universal finite type (or Vassiliev) invariant (see Altschuler-Freidel [1] and Thurston [16]).

There is a higher dimensional analogue of these theories with a certain variant of Jacobi diagrams, that is, isotopy invariants of long higher dimensional embeddings $\mathbb{R}^{n} \rightarrow \mathbb{R}^{n+2}$ (long $n$-knots). The suitable diagrams for higher dimensions are graphs with two kinds of edges corresponding to the "angular forms" in $\mathbb{R}^{n+2}$ and in $\mathbb{R}^{n}$ respectively. The diagrams have degrees given by half the number of vertices. In his seminal paper, Bott [4] constructed an invariant for higher dimensional knots associated to degree 2 diagrams. After that Cattaneo and Rossi [7; 15] gave a path integral formula 
for invariants of higher dimensional embedded manifolds into a manifold and, as a perturbative expansion, they obtained invariants for higher dimensional knots, which will be denoted by $z_{k}$ in this paper, associated to degree- $k$ diagrams. It may be an analogue of the 3-dimensional perturbative Chern-Simons theory and Bott's invariant is the degree 2 part of Cattaneo-Rossi's invariant. So we call $z_{k}$ the Bott-Cattaneo-Rossi invariant, or the BCR invariant for short. More precisely, Cattaneo and Rossi proved that $z_{k}$ (or its framing correction) is an isotopy invariant if $n$ is odd (or $(n, k)=(2,3)$ ). The BCR invariant currently seems to be a new invariant of long $n$-knots. However, it has not been known whether $z_{k}$ is nontrivial or not. We try to understand its features, in particular, whether there is some connection with known invariants.

In this paper we study the invariant $z_{k}$ restricting to a certain class of long $n$-knots called long ribbon $n$-knots. The class of long ribbon $n-$ knots is a rather familiar class known to have similarities to the classical knot theory in 3-dimension. In particular, Habiro, Kanenobu and Shima [10] introduced the notion of finite type invariant of ribbon 2-knots, which is straightforwardly generalizable to long ribbon $n$-knots, and showed that the coefficients of the Alexander polynomial expanded as a power series in $t-1$, are finite type invariants. Finite type invariant of type $k$ is defined by the condition that a given invariant vanishes at any ' $(k+1)$ th order differential' of a ribbon 2-knot. Moreover, Habiro and Shima [11] obtained a remarkable result saying that the set of all finite type invariants is isomorphic as a graded algebra to the polynomial algebra in the coefficients of the Alexander polynomial.

Restricting to long ribbon $n$-knots, we characterize the BCR invariant $z_{k}$ as a finite type invariant (Theorem 4.1). In particular, we determine its 'highest order term' explicitly. As a consequence, we obtain a description in terms of the coefficients of the logarithm of the Alexander polynomial at $t=e^{h}$ with an explicit highest order term. In particular, it shows that the BCR invariant is nontrivial. The proof is done by choosing an embedding for a long ribbon $n-k n o t$ in some extreme situation and then computing $z_{k}$ explicitly at the $k$ th order differentials of long ribbon $n$-knots. In the limiting situation, the computation of the integral is highly reduced and we can compute explicitly. The presence of two kinds of edges in the diagrams appearing in the definition of the BCR invariant makes the computation slightly complicated. But the spirit in the computation is similar to that of Altschuler and Friedel [1], and of Thurston [16].

This paper is organized as follows. In Section 2, the definition of the BCR invariant $z_{k}$ is given and the Cattaneo-Rossi's result concerning the invariance of their invariant is stated. The BCR invariant is defined as a linear combination of the integrals over the configuration spaces associated with certain graphs. In Section 3, we will explain the generality on the space of graphs from which each coefficient in $z_{k}$ at a configuration 
space integral of a graph are determined. This section is somewhat digressive but needed in the invariance proof of $z_{k}$ in Appendix A. In Section 4, we restrict the study to long ribbon $n$-knots. The notion of Habiro, Kanenobu and Shima's finite type invariant is recalled and the main theorem (Theorem 4.1) about the characterization of $z_{k}$ as a finite type invariant is stated and proved. The relation between $z_{k}$ and the Alexander polynomial is explained at the end of Section 4. Appendix A is devoted to the self-contained proof of invariance of the BCR invariant $z_{k}$, filling in the details not explicitly described by Rossi [15].

Acknowledgments The author would like to thank his adviser Professor Tomotada Ohtsuki for helpful comments, careful reading of a manuscript and encouragements. The author would also like to thank Professors Alberto Cattaneo, Kazuo Habiro, Toshitake Kohno, Christine Lescop, Carlo Rossi and referee for reading an earlier version of this paper, pointing out some errors, and for helpful suggestions. Especially, Carlo Rossi helped the author to understand their proof of invariance by correcting the author's misunderstandings and by providing his thesis.

\section{Invariants of long $n$-knots}

A long $n-k n o t$ is the image of a smooth long embedding $\psi$ of $\mathbb{R}^{n}$ into $\mathbb{R}^{n+2}$ that is standard near $\infty$, that is, we assume that there exists an $n$-ball $D \subset \mathbb{R}^{n}$ such that $\psi\left|\left(\mathbb{R}^{n} \backslash D\right)=i\right|\left(\mathbb{R}^{n} \backslash D\right)$ where $i: \mathbb{R}^{n} \hookrightarrow \mathbb{R}^{n+2}$ is the natural inclusion.

In this section, we shall review the definition of the BCR invariant, which is an isotopy invariant of long $n$-knots. We review the definitions of

- Jacobi diagrams and weights $w_{k}$ of them, and

- configuration space integral associated to a Jacobi diagram,

and define the BCR invariant as a linear combination of configuration space integrals for diagrams whose coefficients are their weights.

\subsection{Jacobi diagrams}

A Jacobi diagram is an oriented graph $\Gamma$ valence at most 3 with two kinds of edges, $\theta$-edges (depicted by directed dashed lines) and $\eta$-edges (depicted by directed solid lines) such that:

- The admissible combinations of incident oriented edges to a vertex are: 


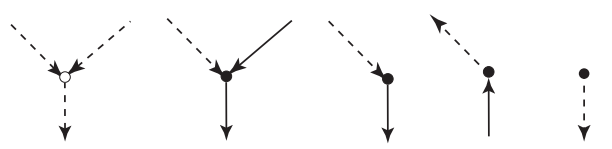

We will call a trivalent vertex with three incident $\theta$-edges an external vertex and in pictures we depict such a vertex by a white dot. We will call a non-external vertex an internal vertex and in pictures we denote it by a black dot.

- $\Gamma$ does not have a subgraph that looks like one of the following forms:

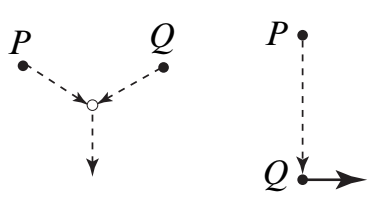

where no other edges are ingoing to both $P$ and $Q$.

If $\Gamma$ does not have subgraphs as in (1) and if $\Gamma$ is connected, then the form of $\Gamma$ is rather restricted: it must be a cyclic alternating sequence of the following two paths with $\theta$-edges stuck into:

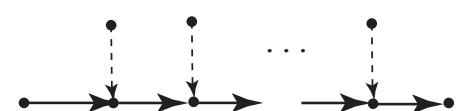

(a)

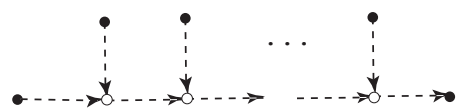

(b)

Here we allow a path to be cyclic. We will call a $\theta$ edge stuck into the path a hair. In particular, any such diagram includes just one cycle. The following picture is a typical example of this observation:

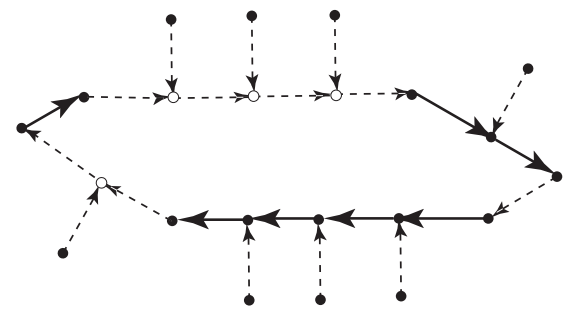

A vertex orientation of a Jacobi diagram $\Gamma$ is a choice of orderings of two ingoing $\theta$-edges incident to each external vertex modulo even number of swappings. In order to represent vertex orientations, we assume that diagrams may be depicted so that the three incident $\theta$-edges

(the outgoing edge of $v$, first incident $\theta$-edge, second incident $\theta$-edge) are arranged in the anti-clockwise order in a plane diagram. 


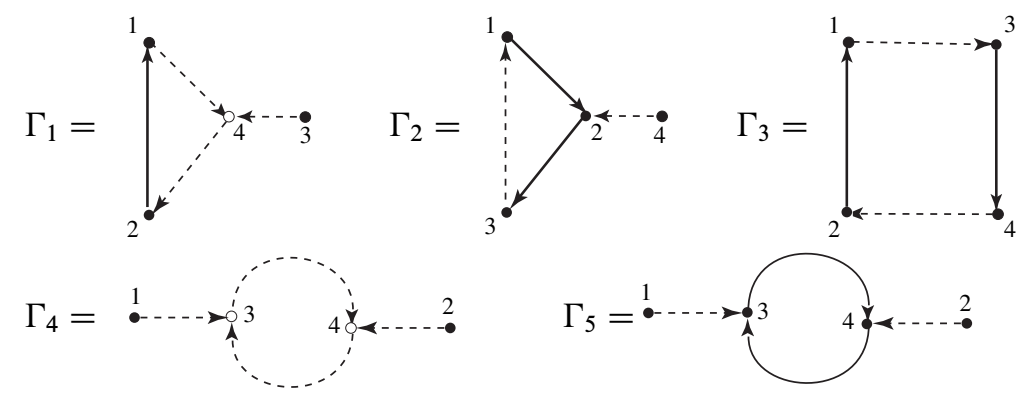

Figure 1: All degree-2 connected Jacobi diagrams up to automorphism

The degree of a Jacobi diagram is defined to be half the number of vertices. The complete list of connected Jacobi diagrams of degree 2 up to automorphism is shown in Figure 1.

An automorphism of a Jacobi diagram is an automorphism $\varphi$ on the set of vertices sending internal vertices to internal vertices (and thus external vertices to external vertices) and inducing a bijection on the set of edges which sends each oriented edge $e=(i, j)$ to an oriented edge $(\varphi(i), \varphi(j))$. We call a $\theta$-edge connecting two internal vertices a chord. We denote the group of automorphisms of $\Gamma$ by Aut $\Gamma$.

2.1.1 The weight function $w_{k}$ on Jacobi diagrams Let $\mathcal{G}_{k}^{0}$ denote the set of all degree- $k$ connected Jacobi diagrams up to automorphism. We will define the invariants of higher dimensional knots in Section 2.3 whose values are $\mathbb{R}$-linear combinations of the weight function $w_{k}$ on Jacobi diagrams, namely, a certain map $w_{k}: \mathcal{G}_{k}^{0} \rightarrow \mathbb{R}$, so that their values are in $\mathbb{R}$. This map $w_{k}$ is defined as follows.

We associate a sign with each trivalent vertex (not only external vertices) by the following rule:
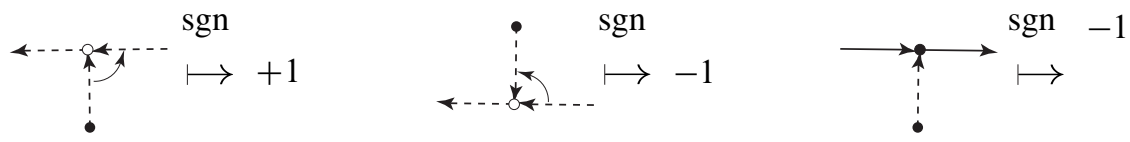

and set

$$
w_{k}(\Gamma)=\prod_{v \text { trivalent }} \operatorname{sgn}(v)
$$

for any $\Gamma$. 


\subsection{Configuration space}

Let $\psi: \mathbb{R}^{n} \rightarrow \mathbb{R}^{n+2}$ be a smooth embedding for a long $n$-knot which is standard near $\infty$. We consider that $\mathbb{R}^{n+2}$ is the complement of a fixed point $\infty$ in $S^{n+2}$.

Let $\Gamma$ be a Jacobi diagram with $q$ internal and $s$ external vertices. The configuration space associated with $(\Gamma, \psi)$ denoted by $C_{\Gamma}^{0}(\psi)$ is defined by

$$
\begin{gathered}
C_{\Gamma}^{0}(\psi):=\left\{\left(x_{1}, \ldots, x_{q} ; x_{q+1}, \ldots x_{q+s}\right) \mid\right. \\
x_{1}, \ldots, x_{q} \in \mathbb{R}^{n}, x_{q+1}, \ldots, x_{q+s} \in \mathbb{R}^{n+2} ; \\
x_{i} \neq x_{j} \quad \text { if } i, j \geq q+1, i \neq j ; \\
\psi\left(x_{i}\right) \neq \psi\left(x_{j}\right) \quad \text { if } i, j \leq q, i \neq j ; \\
\left.\psi\left(x_{i}\right) \neq x_{j} \quad \text { if } i \leq q, j \geq q+1\right\} .
\end{gathered}
$$

To see the convergence of the integral over $C_{\Gamma}^{0}$, we use the compactification $C_{\Gamma}(\psi)$ of $C_{\Gamma}^{0}(\psi)$ used by Axelrod-Singer [2] and Bott-Taubes [5], in analogy of FultonMacPherson [8], which is a smooth manifold with corners. Namely, $C_{\Gamma}$ is obtained by a sequence of blow-ups along all the diagonals (see Section A.1) and along the infinity, in the way described in [5]. Detailed descriptions of the compactification used in this paper are found in Rossi [15]. We remark that there is a natural diffeomorphism between $\operatorname{int}\left(C_{\Gamma}\right)$ and $C_{\Gamma}^{0}$.

\subsection{Integral over configuration space}

Let $E_{\theta}(\Gamma)$ be the set of $\theta$-edges that

(1) are included in the cycle of $\Gamma$, or

(2) end at an internal vertex.

The embedding $\psi: \mathbb{R}^{n} \rightarrow \mathbb{R}^{n+2}$ determines an embedding of a configuration of points on $\mathbb{R}^{n}$ into that of points on $\mathbb{R}^{n+2}$ in $C_{\Gamma}$ by setting $\psi\left(x_{q+i}\right)=x_{q+i}(i \geq 1)$. For each $\theta$-edge $e=(i, j)$ of $\Gamma$ (and each $\eta$-edge $f=(k, l)$ of $\Gamma)$, let $\theta_{e}:=u\left(\psi\left(x_{j}\right)-\right.$ $\left.\psi\left(x_{i}\right)\right)^{*} \omega_{n+1}$ (and $\left.\eta_{f}:=u^{\prime}\left(x_{k}-x_{l}\right)^{*} \omega_{n-1}\right)$ be the $(n+1)$-form on the 2-point configuration space $C_{e}$ (resp. the $(n-1)$-form on $C_{f}$ ), where $\omega_{p}$ is the $S O(p+1)$ invariant unit volume form on $S^{p}$, and the Gauss map $u: \mathbb{R}^{n+2} \backslash\{0\} \rightarrow S^{n+1}$ (and $\left.u^{\prime}: \mathbb{R}^{n} \backslash\{0\} \rightarrow S^{n-1}\right)$ is defined by

$$
u(x)=\frac{x}{\|x\|}, \quad u^{\prime}(x)=\frac{x}{\|x\|} .
$$

Algebraic $8 \mathcal{G}$ Geometric Topology, Volume 7 (2007) 
We define a differential form on $C_{\Gamma}(\psi)$ by

$$
\begin{aligned}
& \omega(\Gamma):= \bigwedge_{e \in E_{\theta}(\Gamma)} \omega(e), \\
& \omega(e):= \begin{cases}\theta_{e} \wedge \eta_{f} & \text { if the } \eta \text {-edge } f \text { goes out from the target of } e \\
\theta_{e} \wedge \theta_{f} & \text { if the } \theta \text {-edge } f \text { (a hair) ends at the target of } e \\
& \text { with the vertex orientation determined by }(e, f) \\
\theta_{f} \wedge \theta_{e} & \text { if the } \theta \text {-edge } f \text { (a hair) ends at the target of } e \\
\text { with the vertex orientation determined by }(f, e)\end{cases}
\end{aligned}
$$

Note that $\omega(\Gamma)$ is of the form $\pm \prod_{e} \theta$-edges of $\Gamma \theta_{e} \prod_{f} \eta$-edges of $\Gamma \eta_{f}$. When $n$ is odd, the sign of the form is always + because all $\theta$ - and $\eta$-forms have even degree. When $n$ is even, both signs may occur.

Let $\Omega$ be an orientation of $C_{\Gamma}(\psi)$. We define

$$
I(\Gamma, \Omega):=\int_{C_{\Gamma}(\psi)} \omega(\Gamma),
$$

in which the orientation of $C_{\Gamma}$ is given by $\Omega$. Note that if $n$ is even, the configuration space can be canonically oriented as a local product of even-dimensional oriented manifolds. Let

$$
I(\Gamma):=I(\Gamma, \Omega(\Gamma)),
$$

where $\Omega(\Gamma)$ is defined in Lemma 2.1 below if $n$ is odd.

Lemma 2.1 Let $n$ be an odd integer $\geq 3$. Let $\Gamma$ be a vertex oriented Jacobi diagram and let $\bar{\Gamma}$ be $\Gamma$ with its vertex orientation at one vertex reversed. Then there exists a choice of orientation $\Omega=\Omega(\Gamma)$ on $C_{\Gamma}(\psi)$ such that

$$
\Omega(\bar{\Gamma})=-\Omega(\Gamma)
$$

Proof Let $\left(X^{1}, \ldots, X^{n+2}\right) \in \mathbb{R}^{n+2}$ be the coordinate of an external vertex of $\Gamma$ and let $\left(X^{1}, \ldots, X^{n}\right) \in \mathbb{R}^{n}$ be the coordinate of an internal vertex of $\Gamma$. Decompose each $\theta$-edge into two half edges. To each half edge $\bar{e}$, we associate an odd form $\Omega_{\bar{e}}$ which are combined into an orientation of $C_{\Gamma}$ as follows.

If $\bar{e}$ is one of three half edges meeting at an external vertex $v$ with the vertex orientation given by a bijection $o_{v}$ : $\{$ the two ingoing half edges $\} \rightarrow\{1,2\}$, set

$$
\Omega_{\bar{e}}:= \begin{cases}d X_{v}^{o_{v}} & \text { if } \bar{e} \text { is ingoing and } o_{v}(\bar{e})=1,2 \\ d X_{v}^{3} \wedge \ldots \wedge d X_{v}^{n+2} & \text { if } \bar{e} \text { is outgoing }\end{cases}
$$


If $\bar{e}$ is a half edge meeting an internal vertex, then set

$$
\Omega_{\bar{e}}:=d X_{v}^{1} \wedge \ldots \wedge d X_{v}^{n} .
$$

In terms of this, we define $\Omega_{e}:=\Omega_{\bar{e}_{+}} \wedge \Omega_{\bar{e}_{-}}$for each $\theta$-edge $e$ with $e=\left(\bar{e}_{-}, \bar{e}_{+}\right)$ and define

$$
\Omega(\Gamma):=\bigwedge_{e \in E_{\theta}(\Gamma)} \Omega_{e}
$$

Then the property (3) is because both $\Omega_{\bar{e}}$ and $\Omega_{\bar{e}^{\prime}}$ are of odd degrees, and

$$
\left(d X_{v}^{1} \wedge \Omega_{\bar{e}}\right) \wedge\left(d X_{v}^{2} \wedge \Omega_{\bar{e}^{\prime}}\right)=-\left(d X_{v}^{1} \wedge \Omega_{\bar{e}^{\prime}}\right) \wedge\left(d X_{v}^{2} \wedge \Omega_{\bar{e}}\right)
$$

for some half-edges $\bar{e}$ and $\bar{e}^{\prime}$.

Further, $\omega(\bar{\Gamma})=(-1)^{n+1} \omega(\Gamma)$ because

$$
\theta_{e} \wedge \theta_{f}=(-1)^{n+1} \theta_{f} \wedge \theta_{e}
$$

This together with Lemma 2.1 implies

$$
I(\bar{\Gamma}, \Omega(\bar{\Gamma}))=-I(\Gamma, \Omega(\Gamma))
$$

for any $n$.

It follows from the definition of $w_{k}$ that $w_{k}(\bar{\Gamma})=-w_{k}(\Gamma)$. Therefore the product $I(\Gamma) w_{k}(\Gamma)$ does not depend on the vertex orientation of $\Gamma$. But it is still sensitive under a reversion of the orientation of an edge. This is the reason why we fix the edge-orientation.

\subsection{Invariants of long $n$-knots}

For $k \geq 2$ and an embedding $\psi: \mathbb{R}^{n} \rightarrow \mathbb{R}^{n+2}$, let

$$
z_{k}(\psi):=\frac{1}{2} \sum_{\Gamma \in \mathcal{G}_{k}^{0}} \frac{I(\Gamma)(\psi) w_{k}(\Gamma)}{|\operatorname{Aut} \Gamma|} \in \mathbb{R}
$$

where $\mid$ Aut $\Gamma \mid$ denotes the order of the group Aut $\Gamma$. Recall that $\mathcal{G}_{k}^{0}$ is the set of all connected Jacobi diagrams of degree $k$ up to automorphism. Here we assume that each term $I(\Gamma)(\psi) w_{k}(\Gamma)$ is given with any choice of vertex orientation of $\Gamma$.

The tangent of the embedding $\psi$ defines the map

$$
d \psi: \mathbb{R}^{n} \rightarrow I_{n}\left(\mathbb{R}^{n+2}\right),
$$

where $I_{n}\left(\mathbb{R}^{n+2}\right)$ is the space of all linear injective maps $\mathbb{R}^{n} \rightarrow \mathbb{R}^{n+2}$. Namely, $d \psi(x)$ for $x \in \mathbb{R}^{n}$ is defined by the linear part of the local embedding near $x$.

Algebraic $8 \mathcal{G}$ Geometric Topology, Volume 7 (2007) 
Cattaneo and Rossi $[7 ; 15]$ remark that they proved the following theorem.

Theorem 2.2 If $n$ is an odd integer $\geq 3$ and $k \geq 2$, then $z_{k}$ is an isotopy invariant of long $n$-knots.

If $n=2$, then there exists a certain 2-form $\rho$ on $I_{2}\left(\mathbb{R}^{4}\right)$ (defined in Proposition A.12) such that

$$
\widehat{z}_{3}(\psi):=z_{3}(\psi)+\int_{C_{1}}(d \psi)^{*} \rho
$$

is an isotopy invariant of long 2-knots, where $C_{1}:=B l\left(S^{2},\{\infty\}\right)$ denotes the blow-up of $S^{2}$ along $\infty \in S^{2}$.

We give a self-contained proof of Theorem 2.2 in Appendix A. For $n=2$, the vanishing of $z_{2}$ is proved by Rossi [15] (see also Proposition 2.5). So $\widehat{z}_{3}$ is the first possibly nontrivial invariant.

By Theorem 2.2, we may write $z_{k}(K)$ (or $\left.\widehat{z}_{3}(K)\right)$ instead of $z_{k}(\psi)\left(\right.$ resp. $\left.\widehat{z}_{3}(\psi)\right)$ if $K=\operatorname{Im} \psi$. We will call $z_{k}$ the $k$ th BCR invariant.

Example 2.3 From Figure 1 and the weights,

$$
w_{2}\left(\Gamma_{1}\right)=-w_{2}\left(\Gamma_{2}\right)=w_{2}\left(\Gamma_{3}\right)=w_{2}\left(\Gamma_{4}\right)=w_{2}\left(\Gamma_{5}\right)=1,
$$

the degree 2 term $z_{2}$ may be given as follows:

$$
z_{2}=\frac{1}{2} I\left(\Gamma_{1}\right)-\frac{1}{2} I\left(\Gamma_{2}\right)+\frac{1}{4} I\left(\Gamma_{3}\right)
$$

Here, $I\left(\Gamma_{4}\right)=I\left(\Gamma_{5}\right)=0$ because $I\left(\Gamma_{4}\right)$ is the integral of $4(n+1)$-form over $3(n+1)$ dimensional submanifold of the target manifold of the Gauss maps (since the targets of two edges by the map $u$ coincide) and similarly for $I\left(\Gamma_{5}\right)$. We will see here that $(5)$ is equal to $\left(\frac{1}{2}\right.$ times $)$ the second invariant in Cattaneo-Rossi [7], which, in this degree, was first obtained by Bott [4]:

(6) $z_{2}=\frac{1}{2} \int_{C_{3,1}} \theta_{14} \theta_{24} \theta_{34} \eta_{12}-\frac{1}{2} \int_{C_{4,0}} \theta_{13} \theta_{24} \eta_{12} \eta_{34}+\frac{1}{4} \int_{C_{4,0}} \theta_{13} \theta_{24} \eta_{12} \eta_{23}$

\subsection{Vanishing of some terms of $z_{k}$}

By the following proposition, most terms of $z_{k}$ in lower odd degrees vanish in the case $n$ is odd. More precisely, for degrees up to three, the direct proof of Proposition 2.4 is given by Rossi [15] for all individual diagrams. Proposition 2.4 is in some sense a generalization of the arguments in [15]. Let $k$ be the degree of a Jacobi diagram $\Gamma$ and 
$k_{1}, k_{2}, k_{3}\left(k=k_{1}+k_{2}+k_{3}\right)$ be the numbers of the following three parts respectively:

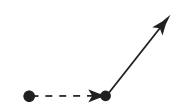

(i)

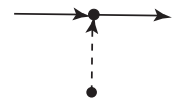

(ii)

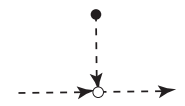

(iii)

Proposition 2.4 Suppose that $\Gamma$ is a vertex oriented Jacobi diagram of degree $k$. Then

$$
I\left(\Gamma^{*}\right) w_{k}\left(\Gamma^{*}\right)= \begin{cases}(-1)^{k} I(\Gamma) w_{k}(\Gamma) & \text { if } n \text { is odd } \\ (-1)^{k-1} I(\Gamma) w_{k}(\Gamma) & \text { if } n \text { is even }\end{cases}
$$

where $\Gamma^{*}$ denotes $\Gamma$ with the orientations of all edges involved in its cycle reversed.

Proof If $n$ is odd, then one can show that

(1) $w_{k}\left(\Gamma^{*}\right)=(-1)^{k_{3}} w_{k}(\Gamma)$.

(2) $\Omega\left(\Gamma^{*}\right)=(-1)^{k_{1}+k_{3}} \Omega(\Gamma)$.

(3) $\omega\left(\Gamma^{*}\right)=(-1)^{k_{2}+k_{3}} \omega(\Gamma)$.

(1) follows from the definition. (2) follows because the number of $\theta$-edges involved in the cycle of $\Gamma$ is $k_{1}+k_{3}$. (3) follows because the number of edges involved in the cycle of $\Gamma$ is $2 k_{1}+k_{2}+k_{3}$. Hence (8) holds.

If $n$ is even, then (8) follows from

(1) $w_{k}\left(\Gamma^{*}\right)=(-1)^{k_{3}} w_{k}(\Gamma)$.

(2) $\Omega\left(\Gamma^{*}\right)=\Omega(\Gamma)$.

(3) $\omega\left(\Gamma^{*}\right)=(-1)^{k_{1}+k_{2}-1} \omega(\Gamma)$.

Here, the changing of the orientations in the cycle permutes the integrand form, which is a product of symmetric odd-degree forms. In the case (iii) in (7), let $a, b, c$ denote the three edges in the middle, left, right respectively in the picture. In $\Gamma$, the integrand form locally defined as $\theta_{a} \theta_{b} \theta_{c}=\left(\theta_{a} \theta_{b}\right) \theta_{c}$, and in $\Gamma^{*}$, the integrand form locally defined as $\theta_{c} \theta_{a} \theta_{b}=\theta_{c}\left(\theta_{a} \theta_{b}\right)$. Hence in a path as in (2)(b), the change of the sign of the integrand form, under the change of the orientations in the cycle, is equal to the change of the sign when the intermediate hairs are removed. Since the part as in (ii) may be read equally as (i) in the integrand form, it is enough to show the identity (3) for the diagram with only the case (i) $k_{1}+k_{2}$ times. In this restricted case, the integrand form is of the form $\theta_{1} \eta_{1} \ldots \theta_{k_{1}+k_{2}} \eta_{k_{1}+k_{2}}$. Then the reversed form is $\theta_{1} \eta_{k_{1}+k_{2}} \theta_{k_{1}+k_{2}} \ldots \eta_{1}=(-1)^{k_{1}+k_{2}-1} \theta_{1} \eta_{1} \ldots \theta_{k_{1}+k_{2}} \eta_{k_{1}+k_{2}}$. 
Proposition 2.4 implies the following proposition.

Proposition 2.5 If $n$ is odd, then $z_{k}=0$ for $k$ odd. If $n$ is even, then $z_{k}=0$ for $k$ even.*

\section{The space of Jacobi diagrams}

We shall see that the weight function $w_{k}: \mathcal{G}_{k}^{0} \rightarrow \mathbb{R}$ satisfies some axioms, which are needed in the proof of Theorem 2.2 and see that $w_{k}$ may arise naturally from the generality on some space of Jacobi diagrams in which the axioms are satisfied. In three dimensions, the graph valued perturbative invariant is very strong in the sense that it yields a lot of scalar valued invariants by composing some weight functions on graphs. But in higher dimensions, the graph valued one is not so strong than a scalar valued one, as observed in this section.

Let $\mathbb{R} \mathcal{G}_{k}^{0}$ denote the vector space spanned by elements of $\mathcal{G}_{k}^{0}$. We define $S T, S U, S T 2$, $C$ relations on $\mathbb{R} \mathcal{G}_{k}^{0}$ as follows ${ }^{\dagger}$ :

(9)

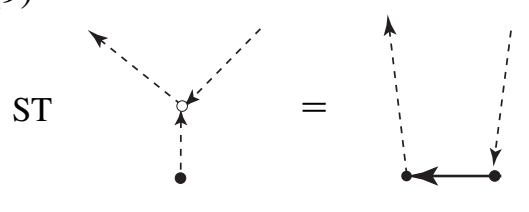

SU
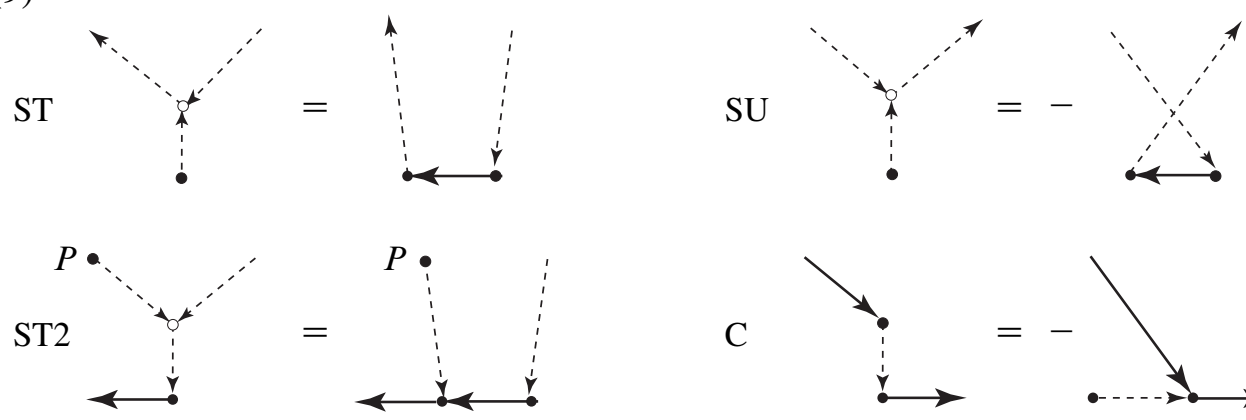

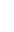


Chern-Simons theory, one could define a graph valued series

$$
z_{k}^{*}(\psi):=\frac{1}{2} \sum_{\Gamma \in \mathcal{G}_{k}^{0}} \frac{I(\Gamma)(\psi)[\Gamma]}{|\operatorname{Aut} \Gamma|} \in \mathcal{A}_{k} .
$$

Since the dimension of $\mathcal{A}_{k}$ is one as proved in Proposition 3.1 below, any linear map $\mathcal{A}_{k} \rightarrow \mathbb{R}$ is a scalar multiple of $w_{k}$, linearly extended to $\mathcal{A}_{k}$ (well-definedness of this extension is proved also in Proposition 3.1). Thus, we obtain the identity

$$
z_{k}^{*}(\psi)=z_{k}(\psi)[\Gamma], z_{k}(\psi)=w_{k} \circ z_{k}^{*}(\psi)
$$

for some $[\Gamma] \in \mathcal{A}_{k}$. Therefore, the BCR invariant is equivalent to the graph valued one.

The vector space structure on $\mathcal{A}_{k}$ is determined as in the following propositions.

Proposition 3.1 (1) The $\theta$ part of a Jacobi diagram forms a disjoint union of chords, trees and wheels. Here wheels are Jacobi diagrams of the following forms:

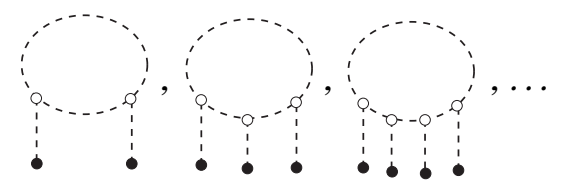

(2) Extend $w_{k}$ linearly to a map $w_{k}: \mathbb{R} \mathcal{G}_{k}^{0} \rightarrow \mathbb{R}$. Then the map $w_{k}: \mathbb{R} \mathcal{G}_{k}^{0} \rightarrow \mathbb{R}$ descends to a well defined $\mathbb{R}$-linear map $w_{k}: \mathcal{A}_{k} \rightarrow \mathbb{R}$ which spans $\mathcal{A}_{k}^{*}$.

(3) The dimension of $\mathcal{A}_{k}$ is 1 for each $k \geq 2$.

Proof (1) immediately follows from the edge-orientation condition of external vertices.

(2) follows from a direct check of the fact that $w_{k}$ satisfies the relations in (9).

For (3), observe first that any Jacobi diagram can be transformed into a Jacobi diagram without $\eta$-edges by ST, SU, C relations. The resulting graph must be a wheel-like graph. So the dimension of $\mathcal{A}_{k}$ is at most one. By (2), $w_{k}$ descends to a nontrivial linear map $w_{k}: \mathcal{A}_{k} \rightarrow \mathbb{R}$, so the dimension of $\mathcal{A}_{k}$ is at least one. Therefore (3) follows.

\section{BCR invariant for long ribbon $n$-knots}

We restrict our study to a certain class of long $n$-knots called long ribbon $n$-knots and characterize the BCR invariant as a finite type invariant in the sense of HabiroKanenobu-Shima [10]. By this characterization, we obtain a description of the BCR invariant in terms of the Alexander polynomial.

Algebraic 8 Geometric Topology, Volume 7 (2007) 


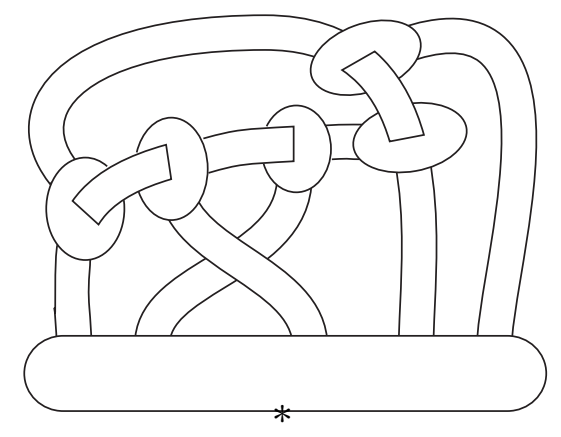

Figure 2: A ribbon presentation

\subsection{Long ribbon $n$-knots and ribbon presentations}

4.1.1 Long ribbon $n$-knots A long ribbon $(n+1)$-disk is the image of an immersion $f$ of a lower half $(n+1)$-plane $D_{-}^{n+1}=\left\{\left(x_{1}, \ldots, x_{n+1}\right) \in \mathbb{R}^{n+1} \mid x_{n+1} \leq 0\right\}$ into $\mathbb{R}^{n+2}$ such that the singularity of $f$ consists of finitely many ribbon singularities and such that $f$ is standard outside a sufficiently large $(n+2)$-ball in $\mathbb{R}^{n+1}$ whose center is the origin. Here a ribbon singularity is an $n$-disk consisting of transverse double points and whose pre-image consists of a proper $n$-disk in $D_{-}^{n+1}$ and an $n$-disk in the interior of $D_{-}^{n+1}$. A long ribbon $n-k n o t$ is a long $n-k n o t$ bounding a long ribbon $(n+1)$-disk.

4.1.2 Ribbon presentations We use ribbon presentations to present long ribbon $n-$ knots. A ribbon presentation $P=\mathcal{D} \cup \mathcal{B}$ in $\mathbb{R}^{3}$ is an immersed oriented 2-disk into $\mathbb{R}^{3}$ with a base point on its boundary having a decomposition consists of $p+1$ disjoint embedded 2-disks $\mathcal{D}=D_{0} \sqcup D_{1} \sqcup D_{2} \sqcup \ldots \sqcup D_{p}$ and $p$ disjoint embedded bands $\mathcal{B}=B_{1} \sqcup B_{2} \sqcup \ldots \sqcup B_{p}$ satisfying the following conditions.

(1) The base point is on the boundary of $D_{0}$.

(2) Each band $B_{i}$ transversely intersects the interiors of the disks in $\mathcal{D}$ as in Figure 3(i).

(3) Each end of a band is attached to the boundary of some disk in $\mathcal{D}$.

An example of a ribbon presentation is depicted in Figure 2.

4.1.3 Associating a long ribbon $n$-knot to a ribbon presentation We can construct a ribbon $(n+1)$-disk $V_{P}$ associated to each ribbon presentation $P$ by

$$
V_{P}=N\left(\mathcal{D} \times[-2,2]^{n-1} \cup \mathcal{B} \times[-1,1]^{n-1}\right) \subset \mathbb{R}^{3} \times \mathbb{R}^{n-1},
$$




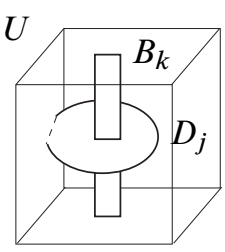

(i)

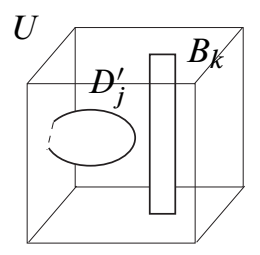

(ii)

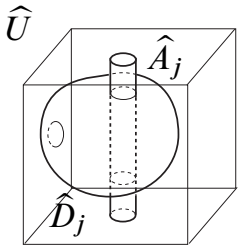

(iii)

Figure 3: Crossing, unclasping

where $N($ ) denotes a smoothing of corners. Then we obtain a long ribbon $n$-knot associated to $P$ by taking the boundary of $V_{P}$ followed by connect summing the standardly embedded plane $\mathbb{R}^{n}$ at the point associated with the base point (the base point may be thickened to form an $(n-1)$-disk. Then define the base point of the $n$-knot to be its center). Note that the connect summing of $\mathbb{R}^{n}$ is not unique even up to isotopy, depending on the position of the base point. We denote by $K_{P}$ the long ribbon $n$-knot associated to a (based) ribbon presentation $P$ and by $\left[K_{P}\right]$ its isotopy class. It is known that any (long) ribbon $n$-knot is isotopic to the one associated to some ribbon presentation.

4.1.4 Crossings Now we give a definition of crossings of both ribbon presentations and long ribbon $n$-knots. For crossings of ribbon presentations, consider a regular neighborhood $U$ of a disk $D_{j} \subset \mathcal{D}$ (of $P=\mathcal{D} \cup \mathcal{B}$ ) including a ribbon singularity inside so that $U \cap P$ consists of

- the disk $D_{j}$,

- a part of some band $B_{k}$ intersecting $D_{j}$.

We call the triple $\left[U, U \cap D_{j}, U \cap B_{k}\right]$ a crossing of $P$ (see Figure 3(i)).

In the associating process of $P \rightsquigarrow K_{P}$ above, $U$ may also be thickened by taking a direct product with $[-3,3]^{n-1}$ to include the associated pieces thickened from $U \cap D_{j}$ and $U \cap B_{k}$. We call the triple $\left(\widehat{U}, \widehat{D}_{j}, \widehat{A}_{j}\right)$ a crossing of $K_{P}$ associated to $\left[U, U \cap D_{j}, U \cap B_{k}\right]$ where

- $\widehat{U}=U \times[-3,3]^{n-1} \subset \mathbb{R}^{n+1}$,

- $\widehat{D}_{j}$ is one of the components of $\widehat{U} \cap K_{P}$ associated to $U \cap D_{j}$,

- $\widehat{A}_{j}$ is one of the components of $\widehat{U} \cap K_{P}$ associated to $U \cap B_{k}$. 
Observe that $\widehat{D}_{j} \cong D^{n}$ and $\widehat{A}_{j} \cong S^{n-1} \times I$. ( $\left.\widehat{U}, \widehat{D}_{j}, \widehat{A}_{j}\right)$ looks like Figure 3(iii). A crossing of a long ribbon $n$-knot isotopic to $K_{P}$ is a triple $\left(\widetilde{U}, \widetilde{D}_{j}, \widetilde{A}_{j}\right),\left(\widetilde{D}_{j} \cup \widetilde{A}_{j} \subset\right.$ $\widetilde{U})$ which is obtained from an associated crossing $\left(\widehat{U}, \widehat{D}_{j}, \widehat{A}_{j}\right)$ on $K_{P}$ as above by an isotopy deforming $K_{P} \cup \widehat{U}$ such that

- it induces an isotopy deforming the trivial link $K_{P} \cap \partial \widehat{U}$ in $\partial \widehat{U}$,

- the number of the components in $K_{P} \cap \partial \widehat{U}$ is preserved during the isotopy,

- it sends $\widehat{D}_{j}$ and $\widehat{A}_{j}$ into $\widetilde{D}_{j}$ and $\widetilde{A}_{j}$ respectively.

4.1.5 Unclasping of a crossing An unclasping of a crossing on a ribbon presentation is defined as a replacement of a crossing $\left[U, U \cap D_{j}, U \cap B_{k}\right]$ in Figure 3(i) with another triple $\left[U, U \cap D_{j}^{\prime}, U \cap B_{k}\right]$ in Figure 3(ii) keeping near $\partial U$ unchanged. It is easy to see that any ribbon presentation can be made into the one without ribbon singularities by a sequence of unclaspings. We can also define an unclasping of a crossing on a long ribbon $n$-knot by applying the associating procedure to both (i) and (ii) in Figure 3 keeping near the boundary unchanged.

4.1.6 Connected sum A connected sum of two long $n$-knots $K \# L$ is defined up to isotopy by arranging $K$ and $L$ along the standard plane $\mathbb{R}^{n} \subset \mathbb{R}^{n+2}$.

\subsection{Finite type invariants of long ribbon $n$-knots}

First we recall the notion of $k$-schemes defined by Habiro-Kanenobu-Shima [10] which is in some sense a higher dimensional analogue of singular knots in Vassiliev's theory of knot invariants.

Let $P=\mathcal{B} \cup \mathcal{D}$ be a based ribbon presentation and let $\left\{c_{1}, \ldots, c_{k}\right\}$ be a set of disjoint crossings on $P$. A $k$-scheme $\left[P ; c_{1}, \ldots, c_{k}\right]$ is defined by

$$
\left[P ; c_{1}, \ldots, c_{k}\right]=\sum_{S \subset\left\{c_{1}, \ldots, c_{k}\right\}}(-1)^{|S|}\left[K_{P S}\right]
$$

where $|S|$ is the size of $S$ and $P^{S}$ is the ribbon presentation obtained from $P$ by unclasping at all the crossings whose labels are in $S$.

Let $\mathcal{J}_{k}$ be the subspace of

$$
\mathcal{L}^{n}:=\operatorname{span}_{\mathbb{R}}\{\text { isotopy classes of long ribbon } n \text {-knots }\}
$$

spanned by all $k$-schemes. It is easy to check that this constitutes a descending filtration on $\mathcal{L}^{n}$ :

$$
\mathcal{L}^{n}=\mathcal{J}_{0} \supset \mathcal{J}_{1} \supset \mathcal{J}_{2} \supset \cdots \supset \mathcal{J}_{k} \supset \cdots
$$

Algebraic 83 Geometric Topology, Volume 7 (2007) 
with the product given by the connected sum. Let $g$ be an $\mathbb{R}$-valued invariant of long ribbon $n$-knots. Then we can extend $g$ naturally to $\mathcal{L}^{n}$ by linearity. We say that $g$ is of type $k$ if it vanishes on $\mathcal{J}_{k+1}$, or equivalently, if $g$ is an element of $\left(\mathcal{L}^{n} / \mathcal{J}_{k+1}\right)^{*}$.

There is a filtration

$$
\mathcal{I}_{0} \subset \mathcal{I}_{1} \subset \mathcal{I}_{2} \subset \cdots \subset \mathcal{I}_{k} \subset \cdots=\mathcal{I}
$$

on the set $\mathcal{I}$ of all $\mathbb{R}$-valued finite type invariants, with $\mathcal{I}_{k}$ being the set of all type $k$ invariants. The two filtrations (10) and (11) are dual to each other in the sense of the following isomorphism, proved by Habiro-Shima [11]:

$$
\mathcal{I}_{k} / \mathcal{I}_{k-1} \cong\left(\mathcal{J}_{k} / \mathcal{J}_{k+1}\right)^{*}
$$

In [11], it is also proved that the elements of the following form constitute a basis

$$
B_{\left(k_{1}, \ldots, k_{r}\right)}:=\left[W_{k_{1}} ; c_{1}, \ldots, c_{k_{1}}\right] \#\left[W_{k_{2}} ; c_{1}, \ldots, c_{k_{2}}\right] \# \ldots \#\left[W_{k_{r}} ; c_{1}, \ldots, c_{k_{r}}\right],
$$

of $\mathcal{J}_{k} / \mathcal{J}_{k+1}$, where $\left[W_{i} ; c_{1}, \ldots, c_{i}\right]$ is defined in Figure 4(a), for possible sequences $\left(k_{1}, \ldots, k_{r}\right)$ of positive integers with $k_{1}+\cdots+k_{r}=k, 2 \leq k_{1} \leq \cdots \leq k_{r}$. We will call the scheme of this form a wheel-like scheme. For $g \in \mathcal{I}_{k}$, we call its projected image in $\mathcal{I}_{k} / \mathcal{I}_{k-1}$ a principal term of $g$, which is in some sense an analogue of highest order term of a polynomial. Then (12) says that the principal term is determined by a linear functional on $\mathcal{J}_{k} / \mathcal{J}_{k+1}$.

We set $\widehat{z}_{k}=z_{k}$ when $n$ is odd and let $\widehat{z}_{3}$ be as in Theorem 2.2 when $n=2$. The main theorem of this paper is stated as follows.

Theorem 4.1 Let $n$ is an odd integer $\geq 3$ and $k \geq 2$, or $(n, k)=(2,3)$. Then

(1) $\widehat{z}_{k}$ is a finite type invariant of type $k$ of long ribbon $n$-knots,

(2) If $n \neq \equiv \bmod 2$, the principal term of $\widehat{z}_{k}$ corresponds to the linear functional $f$ on $\mathcal{J}_{k} / \mathcal{J}_{k+1}$ such that $f\left(B_{(k)}\right)=1$ and $f($ other basis element $)=0$,

(3) If $n \equiv k \bmod 2$, the principal term of $\widehat{z}_{k}$ is zero.

Remark 4.2 Here, although the set of ribbon $n$-knots and the set of long ribbon $n$-knots are different, it can be shown that the filtrations of finite type invariants of both are the same $\$$.

\footnotetext{
$\ddagger$ For instance, any "long" $k$-scheme may be reduced modulo long $(k+1)$-schemes to "wheel-like" ones (see Figure 4). So the graded piece $\operatorname{span}_{\mathbb{R}}\{$ long $k$-schemes $\} / \operatorname{span}_{\mathbb{R}}\{$ long $(k+1)$-schemes $\}$ may be isomorphic to that of usual (non-long) $k$-schemes.
}

Algebraic 83 Geometric Topology, Volume 7 (2007) 


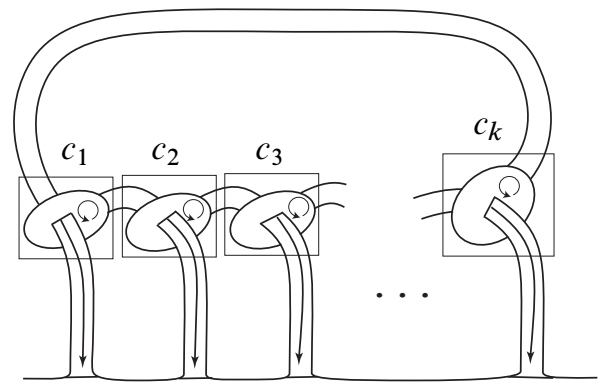

(a)

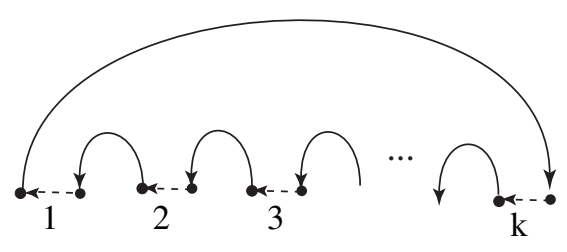

(b)

Figure 4: $\left\{W_{k} ; c_{1}, \ldots, c_{k}\right\}$ and $\Gamma_{k}$

\subsection{Proof of Theorem 4.1}

Let $\left\{P ; c_{1}, \ldots, c_{k}\right\}$ denote a combination of a ribbon presentation $P=\mathcal{D} \cup \mathcal{B}$ and a set of crossings $\left\{c_{1}, \ldots, c_{k}\right\}$ on it. We will call such a combination a $k$-marked ribbon presentation.

4.3.1 Convenient choices of embeddings Assume that $n$ is an odd integer $\geq 3$ and $k \geq 2$ or $(n, k)=(2,3)$. Theorem 4.1 will be proved by computing the values of $\hat{z}_{k}$ for $m$-schemes $\left[P ; c_{1}, \ldots, c_{m}\right]$ with $m \geq k$. Let

$$
\psi^{S}\left(P ; c_{1}, \ldots, c_{m}\right): \mathbb{R}^{n} \rightarrow \mathbb{R}^{n+2}(S \subset\{1, \ldots, m\})
$$

be a choice of an embedding for each $\left[K_{P S}\right]$ which is standard near $\infty$. We will often write $\psi^{S}$ for $\psi^{S}\left(P ; c_{1}, \ldots, c_{m}\right)$ when the marked ribbon presentation $\left\{P ; c_{1}, \ldots, c_{m}\right\}$ considered is understood from the context.

Now we fix some choices of embeddings in the proof, which do not lose the generality because $\widehat{z}_{k}$ is an isotopy invariant, to make the computation simpler. Let $\left[\breve{U}_{i}, \breve{D}_{i}, \breve{A}_{i}\right] \quad(i=1, \ldots, m)$ denote the crossings on $P$ corresponding to $c_{1}, \ldots, c_{m}$ and $\left(U_{i}, D_{i}, A_{i}\right)(i=1, \ldots, m)$ denote the corresponding crossings on $\operatorname{Im} \psi^{\varnothing}$. We choose the embeddings satisfying the following condition, which is made depending on small parameters $\varepsilon>0, \varepsilon_{i}>0(i=1, \ldots, m)$ with $\varepsilon_{i}<\varepsilon$.

(Emb-0) For any $S$ and $i \in\{1, \ldots, m\} \backslash S, \psi^{S}$ and $\psi^{S \cup\{i\}}$ is chosen so that they coincide outside an $(n+2)$-ball in $U_{i}$ with radius $\varepsilon_{i}<\varepsilon$. Indeed, such $\psi^{S}$ and $\psi^{S \cup\{i\}}$ may be obtained by contracting around the center $\left\{\frac{1}{2}\right\} \times S^{n-1} \subset I \times S^{n-1}$ of $A_{i}$ into a very thin cylinder with small $S^{n-1}$ component and let them approach near $D_{i}$. 


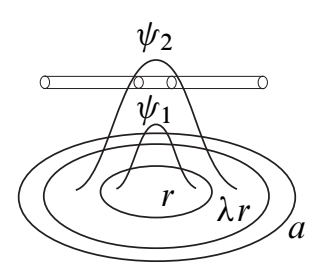

Figure 5: Change of a crossing preserving $d \psi$

This assumption is explained in the lower right of Figure 6. In addition to this assumption, we make another assumption in the following lemma in the case $n=2$ to make the correction term uniformly over the terms of a $k$-scheme. From the procedure of framing correction in this case (see Section A.6.1), the correction term depends only on the map

$$
f=d \psi: \mathbb{R}^{2} \rightarrow I_{2}\left(\mathbb{R}^{4}\right)
$$

giving the tangent 2-framing in $\mathbb{R}^{4}$ of $\psi(x) \in \mathbb{R}^{4}\left(x \in \mathbb{R}^{2}\right)$ where $I_{2}\left(\mathbb{R}^{4}\right)$ is the space of all linear injective maps $\mathbb{R}^{2} \rightarrow \mathbb{R}^{4}$, or the set of 2-framings in $\mathbb{R}^{4}$. The choice of the embeddings in the following lemma can be made compatible with (Emb-0).

Lemma 4.3 Let $P_{1}$ and $P_{2}$ be two ribbon presentations which differ only by one unclasping of a crossing. For long ribbon 2-knots, we can choose certain embeddings $\psi_{i}: \mathbb{R}^{2} \rightarrow \mathbb{R}^{4}, i=1,2$ for the isotopy classes $\left[K_{P_{1}}\right]$ and $\left[K_{P_{2}}\right]$ respectively, so that their tangent 2-frames $f_{i}$ coincide.

Proof We can assume without losing generality that there exist some positive real numbers $r$ and $a$ with $0<r<a$ such that $f_{1}(x)$ is equal to a constant $g \in I_{2}\left(\mathbb{R}^{4}\right)$ in $r \leq\|x\| \leq a$ and such that $f_{1}(x)$ is not a constant function in $\|x\|<r$. Then we can take $\psi_{2}$ as

$$
\psi_{2}(x)= \begin{cases}\lambda \psi_{1}\left(\lambda^{-1} x\right) & \|x\| \leq \lambda r \\ \psi_{1}(x) & \|x\|>\lambda r\end{cases}
$$

for some constant $\lambda$ with $0<\lambda<a / r$. See Figure 5(a) for an explanation of this condition. The naturally induced framing $f_{2}$ for $\psi_{2}$ from $f_{1}$ is

$$
f_{2}(x)= \begin{cases}f_{1}\left(\lambda^{-1} x\right) & \|x\| \leq \lambda r \\ f_{1}(x) & \|x\|>\lambda r\end{cases}
$$

After some change of parametrization in the disk $\|x\| \leq a, f_{2}$ becomes equal to $f_{1}$ without changing the image of $\psi_{2}$. 
We define the formal alternating sum

$$
\psi\left(P ; c_{1}, \ldots, c_{m}\right):=\sum_{S \subset\{1, \ldots, m\}}(-1)^{|S|} \psi^{S} .
$$

Then its configuration space integral $I(\Gamma)$ is given by

$$
I(\Gamma)\left(\psi\left(P ; c_{1}, \ldots, c_{m}\right)\right)=\sum_{S \subset\{1, \ldots, m\}}(-1)^{|S|} I(\Gamma)\left(\psi^{S}\right) .
$$

Proof of Theorem 4.1(1), finiteness of $\widehat{z}_{k} \quad$ Assume that $n$ is an odd integer $\geq 3$ and $k \geq 2$ or $(n, k)=(2,3)$. We will show that $\widehat{z}_{k}\left(\psi\left(P ; c_{1}, \ldots, c_{m}\right)\right)=0$ for any $m$-scheme $\left[P ; c_{1}, \ldots, c_{m}\right]$ with $m>k$, with the choices of embeddings $\psi^{S}$ $(S \subset\{1, \ldots, m\})$ satisfying (Emb-0) and the condition of Lemma 4.3 if $n=2$.

Let $\pi^{S}: C_{\Gamma}\left(\psi^{S}\right) \rightarrow C_{q}\left(\mathbb{R}^{n}\right)$ be the natural projection where $C_{q}\left(\mathbb{R}^{n}\right)$ denotes the configuration space for internal vertices in $\Gamma$. Then $\pi^{S}$ can be considered as a bundle and we can rewrite the integral by the pushforward:

$$
I(\Gamma)\left(\psi^{S}\right)=\int_{C_{\Gamma}\left(\psi^{S}\right)} \omega(\Gamma)\left(\psi^{S}\right)=\int_{C_{q}\left(\mathbb{R}^{n}\right)} \pi_{*}^{S} \omega(\Gamma)\left(\psi^{S}\right)
$$

for some $q n$-form $\pi_{*}^{S} \omega(\Gamma)\left(\psi^{S}\right)$ on $C_{q}\left(\mathbb{R}^{n}\right)$. By using this expression, we can rewrite the alternating sum $I(\Gamma)\left(\psi\left(P ; c_{1}, \ldots, c_{m}\right)\right)$ as an integral of an alternating sum of forms over the common space $C_{q}\left(\mathbb{R}^{n}\right)$ :

$$
I(\Gamma)\left(\psi\left(P ; c_{1}, \ldots, c_{m}\right)\right)=\int_{C_{q}\left(\mathbb{R}^{n}\right)} \sum_{S \subset\{1, \ldots, m\}}(-1)^{|S|} \pi_{*}^{S} \omega(\Gamma)\left(\psi^{S}\right)
$$

Let $V_{1}\left(D_{i}\right)$ be the subset of $C_{q}\left(\mathbb{R}^{n}\right)$ consisting of configurations such that no points are mapped into $D_{i} \subset U_{i}$ by $\psi^{\varnothing}$ (and thus by $\psi^{S}$ ) and $V_{1}\left(A_{i}\right)$ is similarly defined for $A_{i} \subset U_{i}$. Then we have

$$
\int_{V_{1}\left(D_{i}\right) \cup V_{1}\left(A_{i}\right)} \sum_{S \subset\{1, \ldots, m\}}(-1)^{|S|} \pi_{*}^{S} \omega(\Gamma)\left(\psi^{S}\right)=O\left(\varepsilon_{i}\right) .
$$

Here $\varepsilon_{i}<\varepsilon$ is the number taken in (Emb-0) and $O\left(\varepsilon_{i}\right)$ denotes a term which vanishes at the limit $\varepsilon_{i} \rightarrow 0$. (13) is because the integral of the LHS is equal to

$$
\sum_{S \subset\{1, \ldots, \hat{i}, \ldots, m\}}(-1)^{|S|} \int_{V_{1}\left(D_{i}\right) \cup V_{1}\left(A_{i}\right)}\left(\pi_{*}^{S} \omega(\Gamma)\left(\psi^{S}\right)-\pi_{*}^{S \cup\{i\}} \omega(\Gamma)\left(\psi^{S \cup\{i\}}\right)\right)
$$

and this contributes as $O\left(\varepsilon_{i}\right)$ by (Emb-0). 
By (13), the computation reduces to the one for configurations such that both $D_{i}$ and $A_{i}$ includes at least one internal vertices. If $m>k$, this is impossible. So we have $\widehat{z}_{k}\left(\psi\left(P ; c_{1}, \ldots, c_{m}\right)\right)=O(\varepsilon)$ and this must be zero because $\widehat{z}_{k}$ is an invariant by Theorem 2.2. Hence Theorem 4.1(1) is proved.

Proof of Theorem 4.1(2), (3), the principal term of $\widehat{z}_{k} \quad$ Assume that $n$ is an odd integer $\geq 3$ and $k \geq 2$ or $(n, k)=(2,3)$. By Theorem 4.1(1) and by the fact that $\mathcal{J}_{k} / \mathcal{J}_{k+1}$ is generated by wheel-like schemes, we may restrict the computation to wheel-like ones and we assume that $\left\{P ; c_{1}, \ldots, c_{m}\right\}$ is such one. We will call the disk $\breve{D}_{j} \cup B_{j}$, where $\breve{D}_{j}$ is the disk of $P$ in the crossing $c_{j}=\left[\breve{U}_{j}, \breve{D}_{j}, \breve{A}_{j}\right]$ and $B_{j}$ is the band connecting the based disk and $\breve{D}_{j}$, a branch of a marked wheel-like ribbon presentation. Then label the branches of $\left\{P ; c_{1}, \ldots, c_{m}\right\}$ by integers in $\{1, \ldots, m\}$. We choose embeddings $\psi^{S}\left(P ; c_{1}, \ldots, c_{m}\right)$ satisfying the following conditions, which are compatible with (Emb-0) and Lemma 4.3. $\varepsilon>0$ is the same as in (Emb-0).

There are disjoint subsets $\mathrm{D}_{j}$ and $\mathrm{A}_{j}(j=1, \ldots, m)$ of $\mathbb{R}^{n}$ each diffeomorphic to $D^{n}$ and $S^{n-1} \times I$ respectively such that:

(Emb-1) $\quad \psi^{S}=\psi^{\varnothing}$ on $\mathbb{R}^{n} \backslash\left(\bigcup_{j} \mathrm{D}_{j} \cup \bigcup_{j} \mathrm{~A}_{j}\right)$.

(Emb-2) $\quad U_{i} \cap \operatorname{Im} \psi^{S}=\psi^{S}\left(\mathrm{D}_{i} \cup \mathrm{A}_{i}\right)$.

(Emb-3) If the intersection of the $j$ th branch of $P$ and $\breve{U}_{1} \cup \ldots \cup \breve{U}_{m}$ is $\breve{A}_{j_{1}} \cup \breve{D}_{j}$, then

$$
\mathrm{A}_{j_{1}} \cup \mathrm{D}_{j} \subset \mathrm{S}_{j}
$$

where

$$
\mathrm{S}_{j}:=\left\{\left(x_{1}, \ldots, x_{n}\right) \in \mathbb{R}^{n} \mid\left\|x_{1}-j\right\|^{2}+\left\|x_{2}\right\|^{2}+\cdots+\left\|x_{n}\right\|^{2} \leq \varepsilon^{2}\right\} \subset \mathbb{R}^{n} .
$$

(Emb-4) The distance between the crossings $U_{i}$ and $U_{j}$ for $i \neq j$ is very large relative to the diameters of both $U_{i}$ and $U_{j}$. More precisely, the distance is assumed larger than $\frac{1}{\varepsilon} \max \left\{\operatorname{diam} U_{i}\right.$, diam $\left.U_{j}\right\}$.

Figure 6 is a picture explaining these assumptions. One may check that these assumptions are compatible. Note that (Emb-1) and (Emb-4) are the assumptions concerning the image of embeddings and (Emb-2) and (Emb-3) are those concerning the choice of parameterization of $\mathbb{R}^{n}$. Note also that we can not define a limit of the embedding at $\varepsilon=0$ while we can define for the limit $\varepsilon_{i}=0$ (in (Emb-0)).

We prove the following claims with the choices of embeddings $\psi^{S}(S \subset\{1, \ldots, k\})$ satisfying (Emb-0)-(Emb-4) and the condition of Lemma 4.3 if $n=2$.

Claim $1 \widehat{z}_{k}\left(\psi\left(W_{i} ; c_{1}, \ldots, c_{i}\right) \# \psi\left(W_{j} ; c_{1}, \ldots, c_{j}\right)\right)=0$ with $i+j=k, i, j>0$. 


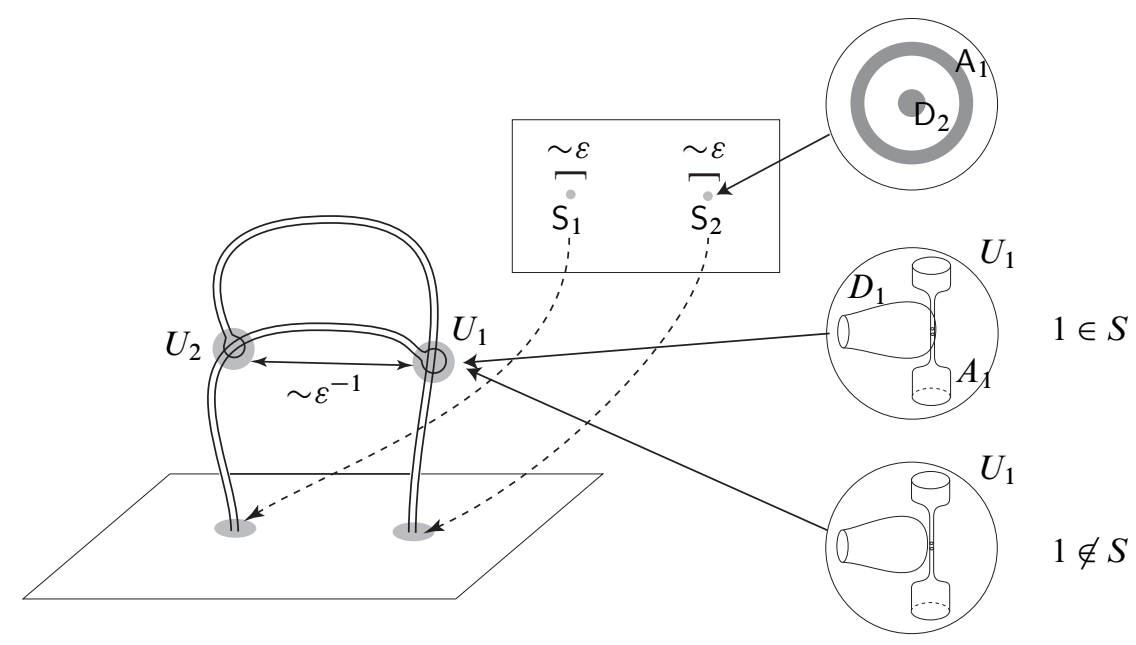

Figure 6: (Emb-0)-(Emb-4)

Claim 2 If $n \not \equiv k \bmod 2, \widehat{z}_{k}\left(\psi\left(W_{k} ; c_{1}, \ldots, c_{k}\right)\right)=w_{k}\left(\Gamma_{k}\right)$ where $\Gamma_{k}$ is defined in Figure 4(b).

Claim 3 If $n \equiv k \bmod 2, \widehat{z}_{k}\left(\psi\left(W_{k} ; c_{1}, \ldots, c_{k}\right)\right)=0$.

The reason for Claim 1-3 to be sufficient to determine the principal term of $z_{k}$ is as follows. Claim 1 implies that $\widehat{z}_{k}$ can be non trivial only on the subspace $\mathcal{P}\left(\mathcal{J}_{k} / \mathcal{J}_{k+1}\right):=\operatorname{span}_{\mathbb{R}}\left\{B_{(k)}\right\}$. So the principal term of $\hat{z}_{k}$ reduces to $\mathcal{P}\left(\mathcal{J}_{k} / \mathcal{J}_{k+1}\right)^{*}$. Thus Claim 2 and 3 characterize the element of $\mathcal{P}\left(\mathcal{J}_{k} / \mathcal{J}_{k+1}\right)^{*}$ for $\widehat{z}_{k}$.

By the proof of Theorem 4.1(1), we may assume $m=k$ to prove Claim 1-3. Proofs of the claims are done by explicit computations of $I(\Gamma)\left(\psi\left(P ; c_{1}, \ldots, c_{k}\right)\right)$ for $\operatorname{deg} \Gamma=k$ and a wheel-like $k$-marked ribbon presentation $\left\{P ; c_{1}, \ldots, c_{k}\right\}$.

Proof of Claim 1 According to the observations in the proof of Theorem 4.1(1), the Jacobi diagrams giving non-zero contribution of $I(\Gamma)\left(\psi\left(P ; c_{1}, \ldots, c_{k}\right)\right)$ are those without external vertices and so we restrict to such Jacobi diagrams in the following. Note that $C_{\Gamma}=C_{q}\left(\mathbb{R}^{n}\right)$ for such $\Gamma$. Let $C_{q}^{\prime}\left(\mathbb{R}^{n}\right)=C_{q}\left(\mathbb{R}^{n}\right) \backslash \bigcup_{i}\left(V_{1}\left(D_{i}\right) \cup V_{1}\left(A_{i}\right)\right)$.

Lemma 4.4 Let $V_{2}(e)$, for $e$ a $\theta$-edge of $\Gamma$, be the set of configurations for $\Gamma$ such that the two ends of $e$ are mapped by the embeddings into some pair of two different balls $U_{i}$ and $U_{j}$ respectively. Then

$$
\int_{V_{2}(e)} \sum_{S \subset\{1, \ldots, m\}}(-1)^{|S|} \omega(\Gamma)\left(\psi^{S}\right)=O(\varepsilon) .
$$

Algebraic $8 \mathcal{G}$ Geometric Topology, Volume 7 (2007) 
Proof of Lemma 4.4 By the assumption (Emb-4), the locus of the image of the relative vector connecting a point in $U_{i}$ and another point in $U_{j}(i \neq j)$ via the Gauss map $u: \mathbb{R}^{n+2} \backslash\{0\} \rightarrow S^{n+1}$ (defined in Section 2.3) for the $\theta$-edge $e$, is included in an arbitrarily small ball embedded into $S^{n+1}$, as $\varepsilon$ tends to 0 . So the integral is $O(\varepsilon)$.

According to Lemma 4.4, only the Jacobi diagrams, each $\theta$-edge of which is entirely mapped into some $U_{i}$, contribute. Since $\Gamma$ is of degree $k$, it follows that such diagrams can not have external vertices.

Lemma 4.5 Let $V_{3}(e)$, for $e$ an $\eta$-edge of $\Gamma$, be the subset of $C_{q}^{\prime}\left(\mathbb{R}^{n}\right)$ consisting of configurations for $\Gamma$ such that two successive internal vertices connected by $e$ split into $\mathrm{S}_{i}$ and $\mathrm{S}_{j}(i \neq j)$. Then

$$
\int_{V_{3}(e)} \sum_{S \subset\{1, \ldots, m\}}(-1)^{|S|} \omega(\Gamma)\left(\psi^{S}\right)=O(\varepsilon) .
$$

Proof of Lemma 4.5 By (Emb-3), the locus of the image of the relative vector connecting the point in $S_{i}$ and another point in some $S_{j}(i \neq j)$ via the Gauss map $u^{\prime}: \mathbb{R}^{n} \backslash\{0\} \rightarrow S^{n-1}$ (defined in Section 2.3) for the $\eta$-edge $e$, is included in an arbitrarily small ball in $S^{n-1}$ as $\varepsilon$ tends to 0 , by the definition of $S_{i}$ 's. So the integral is $O(\varepsilon)$.

For $k$-schemes of the form of connected sum $K_{i} \# K_{j}$ of $i$ - and $j$-schemes with $i+j=k, i, j>1$, any configurations not in $V_{2}(e)$ 's associated to a connected Jacobi diagram, that is, each $\theta$-edge is mapped entirely into some $U_{i}$, must be in some $V_{3}(e)$ because the image of any $\theta$-edge cannot connect $K_{i}$ and $K_{j}$ and then some $\eta$-edge must connect them. Hence Claim 1 is proved.

Proof of Claim 2 Now we compute the precise value of $\widehat{z}_{k}\left(\psi\left(W_{k} ; c_{1}, \ldots, c_{k}\right)\right)$. By Lemma 4.4 and 4.5 , the only contributing term in the sum (4) is the term

$$
\frac{1}{2} \frac{I\left(\Gamma_{k}\right)\left(\psi\left(W_{k} ; c_{1}, \ldots, c_{k}\right)\right) w_{k}\left(\Gamma_{k}\right)}{\mid \text { Aut } \Gamma_{k} \mid} .
$$

By Lemma 4.6 below, this equals

$$
\frac{\mid \text { Aut }^{\prime} \Gamma_{k} \mid w_{k}\left(\Gamma_{k}\right)}{2 \mid \text { Aut } \Gamma_{k} \mid}+O(\varepsilon)=\frac{2 \mid \text { Aut } \Gamma_{k} \mid w_{k}\left(\Gamma_{k}\right)}{2 \mid \text { Aut } \Gamma_{k} \mid}+O(\varepsilon)=w_{k}\left(\Gamma_{k}\right)+O(\varepsilon),
$$

where Aut ${ }^{\prime} \Gamma$ denotes the group of automorphisms of $\Gamma$ considered as an unoriented graph. Roughly, by the above observations, the computation reduces in Lemma 4.6 to the integral over a direct product of some simple spaces and then it is computed as a product of 'linking numbers'. This completes the proof of Claim 2. 
Proof of Claim 3 In the case $n \equiv k \bmod 2$, Claim 3 follows directly from Proposition 2.5.

This completes the proof of Theorem 4.1(2), (3).

Lemma 4.6 Suppose that $n \not \equiv k \bmod 2$. Under the assumptions (Emb-0)-(Emb4), we have $I\left(\Gamma_{k}\right)\left(\psi\left(W_{k} ; c_{1}, \ldots, c_{k}\right)\right)=\mid$ Aut $^{\prime} \Gamma_{k} \mid+O(\varepsilon)$ where Aut' $\Gamma$ denotes the group of automorphisms of $\Gamma$ considered as an unoriented graph.

Proof There are exactly $\mid$ Aut $^{\prime} \Gamma_{k} \mid$ connected components in the reduced configuration space $C_{q}^{\prime}\left(\mathbb{R}^{n}\right) \backslash\left(\bigcup_{e} V_{2}(e) \cup \bigcup_{f} V_{3}(f)\right)$ for $\Gamma_{k}$ each of which is equal up to permutations to

$$
M_{k}:=A_{1} \times D_{1} \times A_{2} \times D_{2} \times \cdots \times A_{k} \times D_{k}
$$

By symmetry, all $\mid$ Aut $^{\prime} \Gamma_{k} \mid$ components contribute as a common value up to sign. Note that in the computation of $I\left(\Gamma_{k}\right)\left(\psi\left(W_{k} ; c_{1}, \ldots, c_{k}\right)\right)$, the integration domain $\mathrm{M}_{k}$ may be assumed to be common for all $S$ because of (Emb-1) and (Emb-2) while the integrand form varies depending on $\psi^{S}$,s.

After a suitable Diff $+\left(\mathbb{R}^{n}\right)$ action on $\mathbb{R}^{n}$ fixing outside $S_{1} \cup \ldots \cup S_{k} \subset \mathbb{R}^{n}$, we may assume in addition to (Emb-0)-(Emb-4) that

\section{(Emb-5)}

$$
\begin{aligned}
\mathrm{A}_{i} & =B^{n}((i+1,0, \ldots, 0) ; 2 \varepsilon / 3) \backslash \operatorname{int} B^{n}((i+1,0, \ldots, 0) ; \varepsilon / 2) \text { for } 1 \leq i<k, \\
\mathrm{~A}_{k} & =B^{n}((1,0, \ldots, 0) ; 2 \varepsilon / 3) \backslash \operatorname{int} B^{n}((1,0, \ldots, 0) ; \varepsilon / 2), \\
\mathrm{D}_{i} & =B^{n}\left((i, 0, \ldots, 0) ; \varepsilon^{2}\right),
\end{aligned}
$$

where $B^{n}(a ; r) \subset \mathbb{R}^{n}$ denotes the $n$-ball with radius $r$ whose center is $a \in \mathbb{R}^{n}$.

This additional assumption can be made so as not to affect the assumptions (Emb-0)(Emb-4) by deforming all $\psi^{S}$ 's for $S \subset\{1, \ldots, k\}$ simultaneously. In the case of $n=2, \mathrm{~S}_{j}, \mathrm{D}_{j}, \mathrm{~A}_{j}$ look like the following picture.

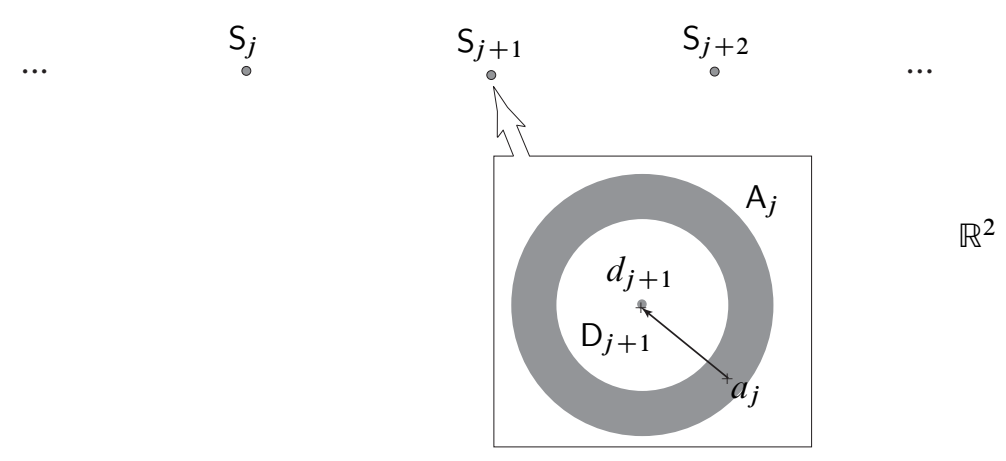

Algebraic $8 \mathcal{G}$ Geometric Topology, Volume 7 (2007) 
Let $\left(a_{1}, d_{1}, \ldots, a_{k}, d_{k}\right)\left(a_{i} \in \mathrm{A}_{i}, d_{i} \in \mathrm{D}_{i}\right)$ be the coordinate of $\mathrm{M}_{k}$. The explicit expression for the LHS integral restricted to $M_{k}$ is

$$
\sum_{S \subset\{1, \ldots, k\}}(-1)^{|S|} \int_{\mathrm{M}_{k}} \bigwedge_{i}\left(u\left(\psi^{S} a_{i}-\psi^{S} d_{i}\right)^{*} \omega_{n+1} \wedge u^{\prime}\left(d_{i+1}-a_{i}\right)^{*} \omega_{n-1}\right) .
$$

It suffices to show that this quantity equals $1+O(\varepsilon)$ because in the case $n \neq \equiv$ mod 2, any automorphism on $\mathrm{M}_{k}$ induced from an automorphism of $\Gamma_{k}$ does not change the orientation of the configuration space and the integrand form. So all $\left|A u t^{\prime} \Gamma_{k}\right|$ connected components contribute as a common value modulo $O(\varepsilon)$.

From the fact that

varying $d_{i}$ fixing all other points only affects (modulo $\left.O(\varepsilon)\right)$ to $u\left(\psi^{S} a_{i}-\right.$ $\left.\psi^{S} d_{i}\right)^{*} \omega_{n+1}$ in $\omega\left(\Gamma_{k}\right)$ because the other form $u^{\prime}\left(d_{i}-a_{i-1}\right)^{*} \omega_{n-1}$, which may also depend on $d_{i}$, contributes as $O(\varepsilon)$ by (Emb-5),

we can write (14) as

$$
\sum_{S \subset\{1, \ldots, k\}}(-1)^{|S|} \int_{\mathrm{M}_{k}} \bigwedge_{i}\left(u\left(\psi^{S} a_{i}-\psi^{S} d_{i}\right)^{*} \omega_{n+1} \wedge u^{\prime}\left(d_{i+1}^{0}-a_{i}\right)^{*} \omega_{n-1}\right)+O(\varepsilon)
$$

where $d_{i+1}^{0}=(i+1,0, \ldots, 0) \in \mathbb{R}^{n}$ for $1 \leq i<k, d_{k+1}^{0}=(1,0, \ldots, 0)$. We will write $\Phi_{i}(\psi):=u\left(\psi a_{i}-\psi d_{i}\right)^{*} \omega_{n+1} \wedge u^{\prime}\left(d_{i+1}^{0}-a_{i}\right)^{*} \omega_{n-1}$ for simplicity bearing in mind that it depends on $a_{i}$ and $d_{i}$. We have

$$
\begin{aligned}
\int_{\mathrm{M}_{k}} \bigwedge_{i} \Phi_{i}\left(\psi^{S}\right)-\int_{\mathrm{M}_{k}} \bigwedge_{i} \Phi_{i}\left(\psi^{S \cup\{j\}}\right) \\
=\int_{\mathrm{M}_{k}(j)} \bigwedge_{i \neq j} \Phi_{i}\left(\psi^{S}\right) \int_{\mathrm{A}_{j} \times \mathrm{D}_{j}}\left(\Phi_{j}\left(\psi^{S}\right)-\Phi_{j}\left(\psi^{S \cup\{j\}}\right)\right)
\end{aligned}
$$

$(S \subset\{1, \ldots, \hat{j}, \ldots, k\})$ where $\mathrm{M}_{k}(j):=\mathrm{A}_{1} \times \mathrm{D}_{1} \times \cdots \times \widehat{\mathrm{A}_{j} \times \mathrm{D}_{j}} \times \cdots \times \mathrm{A}_{k} \times \mathrm{D}_{k}$, because $\Phi_{i}\left(\psi^{S}\right)=\Phi_{i}\left(\psi^{S \cup\{j\}}\right)$ on $\mathrm{M}_{k}(j)$ for $i \neq j$. Thus (14) equals

$$
\begin{gathered}
\sum_{S \subset\{1, \ldots, \hat{j}, \ldots, k\}}(-1)^{|S|} \int_{\mathrm{M}_{k}(j)} \bigwedge_{i \neq j} \Phi_{i}\left(\psi^{S}\right) \int_{\mathrm{A}_{j} \times \mathrm{D}_{j}}\left(\Phi_{j}\left(\psi^{S}\right)-\Phi_{j}\left(\psi^{S \cup\{j\}}\right)\right)+O(\varepsilon) \\
=\left[\int_{\mathrm{A}_{j} \times \mathrm{D}_{j}}\left(\Phi_{j}\left(\psi^{\varnothing}\right)-\Phi_{j}\left(\psi^{\{j\}}\right)\right)\right] \sum_{S \subset\{1, \ldots, \hat{j}, \ldots, k\}}(-1)^{|S|} \int_{\mathrm{M}_{k}(j)} \bigwedge_{i \neq j} \Phi_{i}\left(\psi^{S}\right)+O(\varepsilon) \\
=\prod_{j \in\{1, \ldots, k\}} \int_{\mathrm{A}_{j} \times \mathrm{D}_{j}}\left(\Phi_{j}\left(\psi^{\varnothing}\right)-\Phi_{j}\left(\psi^{\{j\}}\right)\right)+O(\varepsilon) .
\end{gathered}
$$

Algebraic 83 Geometric Topology, Volume 7 (2007) 
Here the fact that $\Phi_{j}\left(\psi^{S}\right)-\Phi_{j}\left(\psi^{S \cup\{j\}}\right)=\Phi_{j}\left(\psi^{\varnothing}\right)-\Phi_{j}\left(\psi^{\{j\}}\right)$ on $\mathrm{A}_{j} \times \mathrm{D}_{j}$ is used. Then by using Lemma 4.7 below $k$ times, we conclude that (14) is $1+O(\varepsilon)$.

\section{Lemma 4.7}

$$
\int_{\mathrm{A}_{j} \times \mathrm{D}_{j}}\left(\Phi_{j}\left(\psi^{\varnothing}\right)-\Phi_{j}\left(\psi^{\{j\}}\right)\right)=1
$$

Roughly, Lemma 4.7 says that the 'linking number' of $D_{j}$ and the axis of $A_{j}$ is 1 .

Proof For each crossing $\left(U_{i}, D_{i}, A_{i}\right)$, consider the three unclaspings of it defined as follows.

$\left(U_{i}, D_{i}^{\prime}, A_{i}\right)$ The result of an unclasping of $\left(U_{i}, D_{i}, A_{i}\right)$ keeping $A_{i}$ fixed.

$\left(U_{i}, D_{i}, A_{i}^{\prime}\right)$ The result of an unclasping of $\left(U_{i}, D_{i}, A_{i}\right)$ keeping $D_{i}$ fixed.

$\left(U_{i}, D_{i}^{\prime}, A_{i}^{\prime}\right)$ Obtained by mixing the $D_{i}^{\prime}$ and $A_{i}^{\prime}$ in the previous two.

Note that these three are isotopic each other and that $\left(U_{i}, D_{i}, A_{i}^{\prime}\right)$ and $\left(U_{i}, D_{i}^{\prime}, A_{i}^{\prime}\right)$ fail to satisfy the condition of Lemma 4.3. However, such replacements do not change the integral, as observed below.

Denote by $\psi\left(D_{j}^{\prime}\right), \psi\left(A_{j}^{\prime}\right), \psi\left(D_{j}^{\prime}, A_{j}^{\prime}\right)$ the corresponding embeddings for the three which are all isotopic to $\psi^{\{j\}}$. Since the three are all isotopic relative to $\partial\left(D_{j} \times A_{j}\right)$ and the integrand form is closed,

$$
\int_{\mathrm{A}_{j} \times \mathrm{D}_{j}} \Phi_{j}\left(\psi\left(D_{j}^{\prime}\right)\right)=\int_{\mathrm{A}_{j} \times \mathrm{D}_{j}} \Phi_{j}\left(\psi\left(A_{j}^{\prime}\right)\right)=\int_{\mathrm{A}_{j} \times \mathrm{D}_{j}} \Phi_{j}\left(\psi\left(D_{j}^{\prime}, A_{j}^{\prime}\right)\right)=\int_{\mathrm{A}_{j} \times \mathrm{D}_{j}} \Phi_{j}\left(\psi^{\{j\}}\right) .
$$

Thus the LHS of the lemma is rewritten as

$$
\int_{\mathrm{A}_{j} \times \mathrm{D}_{j}}\left(\Phi_{j}\left(\psi^{\varnothing}\right)-\Phi_{j}\left(\psi\left(D_{j}^{\prime}\right)\right)-\Phi_{j}\left(\psi\left(A_{j}^{\prime}\right)\right)+\Phi_{j}\left(\psi\left(D_{j}^{\prime}, A_{j}^{\prime}\right)\right)\right)=\int_{\mathrm{A}_{j}^{\circ} \times \mathrm{D}_{j}^{\circ}} \Phi_{j}\left(\psi_{j}^{\circ \circ}\right) \text {. }
$$

Here $\mathrm{X}^{\circ}$ denotes $\mathrm{X} \cup_{\partial}(-\mathrm{X})$ and $\psi_{j}^{\circ \circ}$ is the embedding of $\mathrm{A}_{j}^{\circ} \cup \mathrm{D}_{j}^{\circ}$ into $U_{j} \cup_{\partial}\left(-U_{j}\right) \cong$ $S^{n+2}$ obtained by gluing $\psi(*)$ 's along boundaries.

The computation of the last expression decomposes with respect to the splitting $\mathrm{A}_{j}^{\circ} \cong$ $S^{n-1} \times S^{1}$ as follows:

$$
\begin{aligned}
\int_{\left(v, l, d_{j}\right) \in S^{n-1} \times S^{1} \times \mathrm{D}_{j}^{\circ}} u\left(\psi_{j}^{\circ \circ}(v, l)-\psi_{j}^{\circ \circ}\left(d_{j}\right)\right)^{*} \omega_{n+1} \wedge u^{\prime}\left(d_{j+1}^{0}-(v, l)\right)^{*} \omega_{n-1} \\
=\int_{v \in S^{n-1}}\left(\int_{\mathrm{L}_{j}^{\circ}(v) \times \mathrm{D}_{j}^{\circ}} u\left(\psi_{j}^{\circ \circ}(v, l)-\psi_{j}^{\circ \circ}\left(d_{j}\right)\right)^{*} \omega_{n+1}\right) \wedge \omega_{n-1}(v) .
\end{aligned}
$$

where we use the fact that 
varying $a_{i}$ along the line

$$
\mathrm{L}_{i}(v):=\{(i+1,0, \ldots, 0)+t v \mid t>0\} \cap \mathrm{A}_{i} \subset \mathbb{R}^{n}, v \in S^{n-1}
$$

fixing all other points only affects (modulo $O(\varepsilon))$ to the form $u\left(\psi^{\varnothing} a_{i}-\right.$ $\left.\psi^{\varnothing} d_{i}\right)^{*} \omega_{n+1}$ by $(\mathrm{Emb}-5)$.

To compute the last integral, we choose an orientation of $L_{j}(v)$ given by the direction of $t$ (in the definition of $\mathrm{L}_{j}(v)$ ) increases. Then the induced orientation of $S^{n-1}$ from that of $S^{n-1} \times \mathrm{L}_{j}(\cdot)=\mathrm{A}_{j}$ and from that of $\mathrm{L}_{j}(\cdot)$ differs by $(-1)^{n}$ from the one naturally induced from the orientation of $\mathbb{R}^{n}$ by the outward-normal first convention.

We consider first the integral along $\mathrm{L}_{j}^{\circ}(v) \times \mathrm{D}_{j}^{\circ}$. Here the induced orientation of $S^{n+1}$ by mapping (orientation of $\left.\mathrm{L}_{j}\right) \wedge$ (orientation of $\mathrm{D}_{i}$ ) via $u$ coincides with the one naturally induced from $\mathbb{R}^{n+2}$. To see this, consider the orientation at a point $\left(l_{j}(v), d_{j}\right) \in \mathrm{L}_{j}(v) \times \mathrm{D}_{j}$, identified with a point on the image of the embedding

$$
\begin{aligned}
l_{j}(v) & =\left(p, y_{1}, y_{2}, \ldots, y_{n-1}\right) \in \mathbb{R}^{3} \times[-1,1]^{n-1} \subset \mathbb{R}^{n+2}, \\
d_{j} & =\left(q, y_{1}^{\prime}, y_{2}^{\prime}, \ldots, y_{n-1}^{\prime}\right) \in \mathbb{R}^{3} \times[-2,2]^{n-1} \subset \mathbb{R}^{n+2}
\end{aligned}
$$

where $(p, q)$ are points on the boundary of the two components in a crossing of a ribbon presentation such that the orientation for the factor of $p$ corresponds to the orientation on the band, represented by an oriented arc going forward to the based disk (depicted in Figure 4(a)), and such that the orientation for the factor of $q$ corresponds to the induced orientation on the boundary from the orientation of the ribbon disk. By the definition of the ribbon presentation $W_{k}$ (Figure 4(a)) and the orientation of $\mathrm{L}_{j}(v)$, $d p \wedge d q$ is mapped by the Gauss map into the natural orientation of $S^{2} \subset \mathbb{R}^{3}$ because the linking number of the two arcs is 1 . The orientation of $L_{j}(v) \times D_{j}$ corresponding to varying $p$ fixing $y_{1}, \ldots, y_{n-1}$ (varying along the orientation of $\mathrm{L}_{j}(v)$ ) and varying $d_{j}$, is $d p \wedge d q \wedge d y_{1}^{\prime} \wedge \ldots \wedge d y_{n-1}^{\prime}$. This is mapped by the map $u$ into the natural orientation of $S^{n+1} \subset \mathbb{R}^{n+2}$. Further, the linking number of $\operatorname{Im} \psi_{j}^{\circ \circ} \mid \mathrm{L}_{j}^{\circ}(v)$ and $D_{j}^{\circ}$ is \pm 1 because it is equal to the intersection number of $\operatorname{Im} \psi_{j}^{\circ \circ} \mid \mathrm{L}_{j}^{\circ}(v)$ and an $(n+1)$-disk bounded by $D_{j}^{\circ}$. Thus the integral along $\mathrm{L}_{j}^{\circ}(v) \times \mathrm{D}_{j}^{\circ}$ contributes by 1 .

For the integral along $S^{n-1}$, since the direction of vector from $l_{j}(v)$ to $d_{j+1}^{0}$ coincides with the ingoing normal vector on $S^{n-1}$, the integral contributes by $(-1)^{n} \times(-1)^{n}=1$ and the result follows.

\subsection{Relation with the Alexander polynomial}

The Alexander polynomial $\Delta_{K}(t) \in \mathbb{Z}\left[t, t^{-1}\right]$ for a (long) $n$-knot $K$ is defined by using the Fox calculus for the knot group. See Habiro-Kanenobu-Shima [10] for 
detailed definition. The Alexander polynomial is uniquely determined by the conditions: $\Delta_{K}(1)=1$ and $\left(d \Delta_{K} / d t\right)(1)=0$, and we use such a normalized one. Then a series of invariants $\alpha_{2}, \alpha_{3}, \ldots$ of (long) $n$-knots are defined by

$$
\left.\log \Delta_{K}(t)\right|_{t=e^{h}}=\alpha_{2}(K) h^{2}+\alpha_{3}(K) h^{3}+\cdots \in \mathbb{Q} \llbracket h \rrbracket .
$$

The following result gives a complete correspondence between finite type invariant of ribbon $2-$ knots and $\alpha_{j}$-invariants.

Proposition 4.8 Let $k>1$ and $n>1$. Then

(1) (Habiro-Kanenobu-Shima) $\alpha_{k}$ is a primitive (=additive) invariant of type $k$ of ribbon $n$-knots.

(2) (Habiro-Shima) We have the identification:

$\{\mathbb{Q}$-valued finite type invariants $\}=\mathbb{Q}\left[\alpha_{2}, \alpha_{3}, \ldots\right]$

of graded Hopf algebras.

In Habiro-Shima [11], the results are stated for $n=2$ and it is remarked there that their result is generalized to ribbon $n$-knots. Kazuo Habiro says that the proof for $n>2$ is exactly the same as for $n=2$ and we have checked it. By Remark 4.2, Proposition 4.8 also holds for long ribbon $n$-knots.

Corollary 4.9 Let $n$ be an odd integer $\geq 3$. For long ribbon $n-k n o t s, \widehat{z}_{k}$ is equal to a polynomial in $\alpha_{2}, \alpha_{3}, \ldots, \alpha_{k}\left(\operatorname{deg} \alpha_{j}=j\right)$ of degree $\leq k$ and we have

$$
\widehat{z}_{k} \equiv \begin{cases}-\alpha_{k} & \text { in } \mathcal{I}_{k} / \mathcal{I}_{k-1} \text { if } k \text { is even, } \\ 0 & \text { in } \mathcal{I}_{k} / \mathcal{I}_{k-1} \text { if } k \text { is odd. }\end{cases}
$$

For long ribbon 2-knots, $\widehat{z}_{3}=\alpha_{3}+\lambda \alpha_{2}$ for some constant $\lambda \in \mathbb{R}$.

In particular, Corollary 4.9 implies

Corollary 4.10 The BCR invariant is nontrivial for $(n, k)=($ odd $\geq 3$, even $\geq 2)$ or $(2,3)$.

Proof of Corollary 4.9 Since $\widehat{z}_{k}$ is an invariant of type $k$ by Theorem 4.1(1), $\widehat{z}_{k}$ is a degree at most $k$ polynomial in $\alpha_{2}, \ldots, \alpha_{k}$ by Proposition 4.8.

The principal term of $\hat{z}_{k}$ is determined by Theorem 4.1(2) and Lemma 4.11 below.

Lemma 4.11 The type $k$ invariant $\alpha_{k}$ satisfies the following conditions. 
(1) $\alpha_{k}\left(\mathcal{J}_{i} \# \mathcal{J}_{j}\right)=0$ for $i+j=k, i, j>0$.

(2) $\alpha_{k}\left(\left[W_{k} ; c_{1}, \ldots, c_{k}\right]\right)=(-1)^{k-1}$,

where $W_{k}$ and the crossings $c_{1}, \ldots, c_{k}$ on it are defined in Figure 4(a).

Proof (1) is because $\alpha_{k}$ is additive.

For (2), it suffices to compute the value of $\alpha_{k}$ at $\left[K_{W_{k}}\right]-1$ (1: the class of the trivial knot) because $\left[K_{W_{k}}^{c_{j}}\right]=1$. Since $\left[W_{k}^{c_{1}}\right]=1$ and any other unclasping at $c_{j}(j \neq 1)$ on it yields again the trivial knot, we have $\left[W_{k}^{c_{1}} ; c_{2}, \ldots, c_{k}\right]=0$, and

$$
\begin{aligned}
{\left[W_{k} ; c_{1}, \ldots, c_{k}\right] } & =\left[W_{k} ; c_{2}, c_{3}, \ldots, c_{k}\right]-\left[W_{k}^{c_{1}} ; c_{2}, c_{3}, \ldots, c_{k}\right] \\
& =\left[W_{k} ; c_{2}, c_{3}, \ldots, c_{k}\right]=\left[W_{k}, c_{3}, \ldots, c_{k}\right]-\left[W_{k}^{c_{2}} ; c_{3}, \ldots, c_{k}\right] \\
& =\left[W_{k} ; c_{3}, \ldots, c_{k}\right]=\cdots=\left[W_{k} ; c_{k}\right]=\left[K_{W_{k}}\right]-1 .
\end{aligned}
$$

The fundamental group of $\left[K_{W_{k}}\right]$ is generated by meridians of the part from the based disk and of the parts from the disks $\breve{D}_{j}$ in the crossing $c_{j}$. We denote the former one by $x_{0}$ and the latter ones by $x_{1} \ldots, x_{k}$. Then by the procedure described by Habiro-Kanenobu-Shima [10], the fundamental group of $\left[K_{W_{k}}\right]$ is presented as

$$
\left\langle x_{0}, x_{1}, \ldots, x_{k} \mid x_{1}^{-1} x_{2} x_{0} x_{2}^{-1}, x_{2}^{-1} x_{3} x_{0} x_{3}^{-1}, \ldots, x_{k}^{-1} x_{1} x_{0} x_{1}^{-1}\right\rangle .
$$

The Alexander polynomial of $\left[K_{W_{k}}\right]$ is computed by using Fox differential as follows:

$$
\operatorname{det}\left[\begin{array}{ccccc}
-t^{-1} & t^{-1}-1 & 0 & 0 & \cdots \\
0 & -t^{-1} & t^{-1}-1 & 0 & \cdots \\
0 & 0 & -t^{-1} & t^{-1}-1 & \cdots \\
\vdots & \vdots & \vdots & \vdots & \ddots \\
t^{-1}-1 & 0 & 0 & 0 & \cdots
\end{array}\right]=\left(-t^{-1}\right)^{k}+(-1)^{k-1}\left(t^{-1}-1\right)^{k}
$$

After the normalization, $\Delta_{\left[K_{W_{k}}\right]}(t)=1-(1-t)^{k}$. From this we have

$$
\log \Delta_{\left[K_{W_{k}}\right]}\left(e^{h}\right)=\log \left(1-\left(1-e^{h}\right)^{k}\right)=-(-h)^{k}+o\left(h^{k}\right) .
$$

Hence $\alpha_{k}\left(\left[K_{W_{k}}\right]\right)=(-1)^{k-1}$ and

$$
\alpha_{k}\left(\left[W_{k} ; c_{1}, \ldots, c_{k}\right]\right)=\alpha_{k}\left(\left[K_{W_{k}}\right]-1\right)=\alpha_{k}\left(\left[K_{W_{k}}\right]\right)-\alpha_{k}(1)=(-1)^{k-1}-0 .
$$

\section{Appendix A Invariance proof}

In Cattaneo-Rossi [7] and Rossi [15], the invariance of the higher degree $z_{k}$ is claimed and in some part idea of the proof is given, while explicit proofs are given in the other 
parts even for higher degrees. The explicit descriptions of $z_{k}$ for higher degrees are not given there, while the complete definitions and proofs are given for degrees up to 3. In this section, we give a proof of Theorem 2.2 filling the details in their idea with our definition of $z_{k}$, conventions on the diagrams and the orientations on configuration spaces. We also see that $z_{k}$ can be obtained naturally from a general framework of diagrams and of the geometry of configuration spaces.

The proof of Theorem 2.2 is described in Section A.2. The proof splits into the four cases described in Section A.1.2. More detailed checks corresponding to the four cases, referred in the proof are described in the subsequent subsections starting from Section A.3.

\section{A.1 Descriptions of faces in $\partial C_{\Gamma}$}

A.1.1 Codimension one faces It is known that $C_{\Gamma}$ is a smooth manifold with corners and $\partial C_{\Gamma}$ admits a stratification (see Fulton-MacPherson [8] and Bott-Taubes [5]). We will need only to consider the codimension one faces in $\partial C_{\Gamma}$ for our purpose. So we review here how each codimension one face can be described.

As observed in [8] and Axelrod-Singer [2], the set of codimension one faces in $\partial C_{\Gamma}$ is in bijective correspondence with the set of all subsets of the vertices of $\Gamma$, which are obtained by blowing-up along the corresponding diagonals. Let $\Delta_{A}$ denote the diagonal set consisting of configurations such that all points in $A$ coincide, and let $\Delta_{\infty, A}$ be the diagonal set consisting of configurations such that all points in $A$ coincide at $\infty$. Denote by $\mathcal{S}_{\Gamma, A}$ and $\mathcal{S}_{\Gamma, \infty, A}$ the codimension one faces corresponding to blow-ups along $\Delta_{A}$ and $\Delta_{\infty, A}$ respectively.

Let us briefly see how $\mathcal{S}_{\Gamma, A}$ is presented. Details are found in Rossi [15]. (This is somewhat analogous to the case of knots in 3-dimension. This case is described by Poirier [14] very explicitly.) Let $V(\Gamma)$ denote the set of vertices of $\Gamma$. There are two cases:

Case 1 If $A \subset V(\Gamma)$ does not have internal vertices, then $\mathcal{S}_{\Gamma, A}$ is the pullback bundle $\varphi^{*} B_{A}=B_{A} \times C_{\Gamma / A}$ in the following commutative diagram.

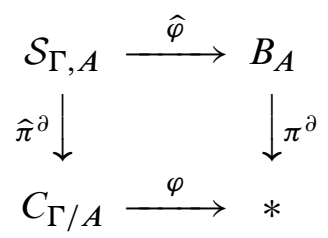

Here 
- $B_{A}$ is the space of configurations of points on $\mathbb{R}^{n+2}$, modulo overall translations and dilations.

- $C_{\Gamma / A}$ is the space of configurations obtained from configurations in $C_{\Gamma}$ by collapsing points in $A$,

- $\pi^{\partial}$ and $\varphi$ are the maps to a point.

We consider each piece in (16) as a fiber over a point in $\operatorname{Emb}\left(\mathbb{R}^{n}, \mathbb{R}^{n+2}\right)$.

Case 2 If $A \subset V(\Gamma)$ has internal vertices, then $\mathcal{S}_{\Gamma, A}$ is the pullback bundle $\varphi^{*} \widehat{B}_{A}$ in the following commutative diagram.

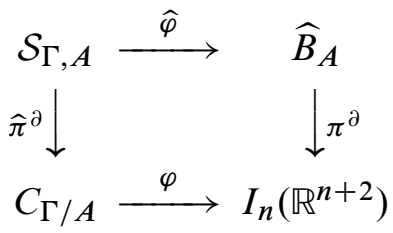

Here

- $\varphi$ is the composition of the embedding and the generalized Gauss map (the tangent map together with an assignment of the $n$-frame in $\mathbb{R}^{n+2}$ determined by the embedding) at the point where the points in $A$ coincide,

- $\widehat{B}_{A}$ is the space of configurations of points on $\left(\mathbb{R}^{n+2}, \iota \mathbb{R}^{n}\right),\left(\iota \in I_{n}\left(\mathbb{R}^{n+2}\right)\right.$ is an $n$-frame in $\mathbb{R}^{n+2}$ ) modulo translations and dilations, together with $\iota \in I_{n}\left(\mathbb{R}^{n+2}\right)$,

- $\pi^{\partial}$ is the map giving the underlying $n$-frame in $\mathbb{R}^{n+2}$.

For the face $\mathcal{S}_{\Gamma, \infty, A}$, the fiber $B_{\infty, A}$ of the unit normal bundle $S N \Delta_{\infty, A}$ over a point in $\Delta_{\infty, A} \subset\left(S^{n+2}\right)^{k}$ where the points in $A$ coincide at $\infty \in S^{n} \subset S^{n+2}$, is identified with the set of points in $T_{\infty} S^{n}$ (resp. $T_{\infty} S^{n+2}$ ) modulo overall translations and dilations along $T_{\infty} S^{n}$. Thus $\mathcal{S}_{\Gamma, \infty, A}$ may be identified with $C_{\Gamma \backslash A} \times B_{\infty, A}$. We will call a point in $B_{A}, \widehat{B}_{A}, B_{\infty, A}$ a relative configuration.

The forms on $C_{\Gamma}^{0}$ defined in Section 2.3 extend smoothly to $C_{\Gamma}$ as the naturally defined forms by Gauss maps via the coordinates determined by the infinitesimal embedding $\iota$.

A.1.2 Classification of codimension one faces Theorem 2.2 will be proved by looking at the integrals restricted to the codimension one faces classified as follows.

Principal faces The faces corresponding to the diagonal where exactly two of the $|V(\Gamma)|$ points coincide in $S^{n+2} \backslash\{\infty\}$. 
Hidden faces The faces corresponding to the diagonal where at least 3 (but not all) of the $|V(\Gamma)|$ points coincide in $S^{n+2} \backslash\{\infty\}$.

Infinite faces The faces corresponding to the diagonal where some of $|V(\Gamma)|$ points coincide at $\infty \in S^{n}$.

Anomalous faces The faces corresponding to the diagonal where all the $|V(\Gamma)|$ points coincide in $S^{n} \backslash\{\infty\}$.

\section{A.2 Proof of Theorem 2.2}

Let $\pi: E \rightarrow B$ be a bundle with $n$ dimensional fiber $F$. Then the push-forward (or integral along the fiber) $\pi_{*} \omega$ of an $(n+p)$-form $\omega$ on $E$ is a $p$-form on $B$ defined by

$$
\int_{c} \pi_{*} \omega=\int_{\pi^{-1}(c)} \omega
$$

where $c$ is a $p$-dimensional chain in $B$.

Let $\pi^{\partial}: \partial_{F} E \rightarrow B$ be the restriction of $\pi$ to $\partial F$-bundle with the orientation induced from $\operatorname{int}(F)$, that is, $\Omega(\partial F)=i_{n} \Omega(F)$ where $n$ is the in-going normal vector field over $\partial F$. Then the generalized Stokes theorem for the pushforward is

$$
d \pi_{*} \omega=\pi_{*} d \omega+(-1)^{\operatorname{deg} \pi_{*}^{\partial} \omega} \pi_{*}^{\partial} \omega .
$$

The derivation of (18) is, for example, in Bott-Taubes [5, Appendix].

Proof of Theorem 2.2 Let $\psi_{0}$ and $\psi_{1}$ be two embeddings $\mathbb{R}^{n} \rightarrow \mathbb{R}^{n+2}$ that are standard near $\infty$. The formula (18) is used to prove the invariance of $z_{k}$ (or $\widehat{z}_{3}$ ) as follows. For each $\Gamma$, consider

$$
\left\{C_{\Gamma}(\psi)\right\}_{\psi \in \operatorname{Emb}\left(\mathbb{R}^{n}, \mathbb{R}^{n+2}\right)} \rightarrow \operatorname{Emb}\left(\mathbb{R}^{n}, \mathbb{R}^{n+2}\right)
$$

as a bundle over the space $\operatorname{Emb}\left(\mathbb{R}^{n}, \mathbb{R}^{n+2}\right)$ of embeddings with fiber the configuration space $C_{\Gamma}$ and consider $z_{k}$ as a 0 -form on $\operatorname{Emb}\left(\mathbb{R}^{n}, \mathbb{R}^{n+2}\right)$, which is defined as a sum of pushforwards of the form $\frac{\omega(\Gamma) w_{k}(\Gamma)}{\mid \text { Aut } \Gamma \mid}$ on the $C_{\Gamma}$-fiber of the bundle (19). Then the invariance of $z_{k}$ relies on the closedness of it because if two embeddings $\psi_{0}$ and $\psi_{1}$ are connected by a smoothly parametrized embeddings $\psi_{t}(t \in[0,1])$, then by Stokes' theorem,

$$
z_{k}\left(\psi_{1}\right)-z_{k}\left(\psi_{0}\right)=\int_{0}^{1} d z_{k}\left(\psi_{t}\right)
$$

Since $\omega(\Gamma)$ is closed,

$$
d I(\Gamma)=\int_{\partial C_{\Gamma}} \omega(\Gamma)
$$

Algebraic 83 Geometric Topology, Volume 7 (2007) 
by (18) where the integral of the RHS is the pushforward restricted to $\partial C_{\Gamma}$ in (19). In particular, only the codimension one faces in $\partial C_{\Gamma}$ contribute to the above integral. Therefore the obstruction to the closedness of $z_{k}$ is

$$
d z_{k}=\sum_{\Gamma} \frac{w_{k}(\Gamma)}{|\operatorname{Aut} \Gamma|} \sum_{S \subset \partial C_{\Gamma: \operatorname{codim} 1}} \int_{S} \omega(\Gamma) .
$$

Recall from A.1.2, there are four types of codimension one faces in $\partial C_{\Gamma}$ : principal faces, hidden faces, infinite faces, anomalous faces.

By Proposition A.1 below, all the principal face contributions cancel each other in the sum. Namely,

$$
\sum_{\Gamma} \frac{w_{k}(\Gamma)}{|\operatorname{Aut} \Gamma|} \sum_{S \subset \partial C_{\Gamma}: \text { principal }} \int_{S} \omega(\Gamma)=0 .
$$

Here the rules of the canceling are given by the ST, ST2 and C relations.

The vanishing of the integral restricted to the hidden and the infinite faces will be discussed in Section A.5 and Section A.6. About the contributions for the anomalous faces will be discussed in Proposition A.12 and A.13.

\section{A.3 Configuration space integral restricted to the faces}

Now we shall see the explicit form of the integral $I(\Gamma)$ extended to the codimension one faces.

In the diagram (16), the form $\omega(\Gamma)$ extended to $\mathcal{S}_{\Gamma, A}$ can be written as

$$
\omega(\Gamma)=\hat{\varphi}^{*} \lambda_{1}\left(\Gamma_{A}\right) \wedge \hat{\pi}^{\partial *} \lambda_{2}(\Gamma / A)
$$

for some $\lambda_{1}\left(\Gamma_{A}\right) \in \Omega^{*}\left(B_{A}\right)$ and $\lambda_{2}(\Gamma / A) \in \Omega^{*}\left(C_{\Gamma / A}\right)$ both determined by graphs and the Gauss maps. Therefore $\hat{\pi}_{*}^{\partial} \omega(\Gamma)=\hat{\pi}_{*}^{\partial} \hat{\varphi}^{*} \lambda_{1}\left(\Gamma_{A}\right) \wedge \lambda_{2}(\Gamma / A)=\varphi^{*} \pi_{*}^{\partial} \lambda_{1}\left(\Gamma_{A}\right) \wedge$ $\lambda_{2}(\Gamma / A)$ by commutativity of (16).

Similarly, in the diagram (17), the form $\omega(\Gamma)$ extended to $\mathcal{S}_{\Gamma, A}$ has the decomposition (21) too for some $\lambda_{1}\left(\Gamma_{A}\right) \in \Omega^{*}\left(\widehat{B}_{A}\right)$ and $\lambda_{2}(\Gamma / A) \in \Omega^{*}\left(C_{\Gamma / A}\right)$ both determined by graphs. $\hat{\pi}_{*}^{\partial} \omega\left(\Gamma_{A}\right)=\varphi^{*} \pi_{*}^{\partial} \lambda_{1}\left(\Gamma_{A}\right) \wedge \lambda_{2}(\Gamma / A)$ also holds.

In other words, in both cases the integral

$$
I(\Gamma, A):=\int_{C_{\Gamma / A}} \varphi^{*} \pi_{*}^{\partial} \lambda_{1}(\Gamma) \wedge \lambda_{2}(\Gamma / A)
$$

along the fiber $C_{\Gamma / A}$, with the orientation induced from $\operatorname{int}\left(C_{\Gamma}\right)$, is precisely the integral of $\omega(\Gamma)$ along the fiber $\mathcal{S}_{\Gamma, A}$, as a fiber over the space of embeddings $\operatorname{Emb}\left(\mathbb{R}^{n}, \mathbb{R}^{n+2}\right)$.

Algebraic $8 \mathcal{G}$ Geometric Topology, Volume 7 (2007) 


\section{A.4 Principal faces}

In this subsection, we will prove the following proposition.

Proposition A.1 For $A \subset V(\Gamma)$ with $|A|=2$,

$$
\sum_{\Gamma \in \mathcal{G}_{k}^{0}} \sum_{A} \frac{I(\Gamma, A) w_{k}(\Gamma)}{|\operatorname{Aut} \Gamma|}=0 .
$$

This proposition shows that the configuration space integrals restricted to principal faces cancel each other.

A.4.1 Quasi Jacobi diagrams Since each principal face corresponds to a collapsing of an edge in a Jacobi diagram, it is represented by using the following graphs obtained by collapsing an edge. We will call such a graph a quasi Jacobi diagram. We say that the vertex where an edge of $\Gamma$ has been collapsed is exceptional. Vertex orientation of a quasi Jacobi diagram is also defined. It is defined as a choice of a bijection

$$
o_{v}:\{\text { two ingoing } \theta \text {-edges incident to } v\} \rightarrow\{1,2\}
$$

for each non-exceptional external vertices $v$ and a bijection

$$
\begin{array}{ll}
o_{v}:\{\text { three ingoing } \theta \text {-edges incident to } v\} \rightarrow\{1,2,3\} & \text { if } v \text { is } 4 \text {-valent } \\
o_{v}:\{\text { two ingoing } \theta \text {-edges incident to } v\} \rightarrow\{1,2\} & \text { otherwise }
\end{array}
$$

for the exceptional vertex $v$ having incident $\theta$-edges.

Let $\Gamma$ be a Jacobi diagram $\mathbb{R}^{n}$ and $e$ be a $\theta$-edge of $\Gamma$. The operator $\delta_{e}$ of $\Gamma$ giving a quasi Jacobi diagram is defined as follows:

(1)

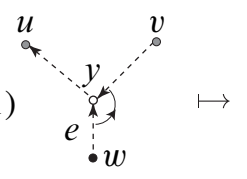

(3)

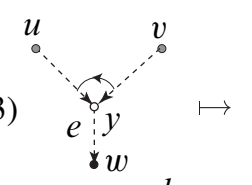

(5)

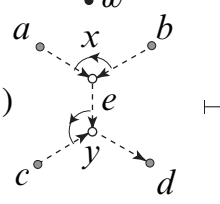

(7)

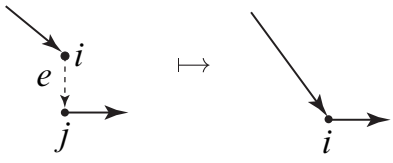

(2)

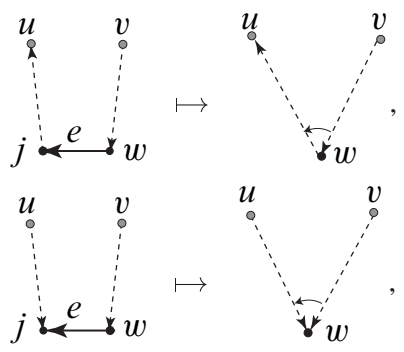

(6)

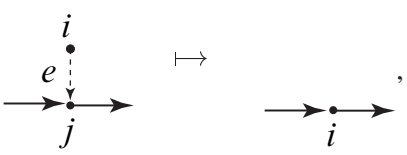


A.4.2 Compactified configuration space for $\delta_{e} \Gamma$ We define the configuration space $C_{\delta_{e} \Gamma}^{0}(\psi)$ so that a point in $C_{\delta_{e} \Gamma}^{0}(\psi)$ represents a position on the diagonal $\Delta_{\{i j\}}$ in $\left(S^{n+2}\right)^{k}$ where $e=(i, j)$. Let $C_{\delta_{e} \Gamma}(\psi)$ be its closure in $C_{\Gamma}(\psi)$. Let

$$
\begin{aligned}
C_{\delta_{e} \Gamma}^{0}(\psi):= & \left\{\left(x_{1}, \ldots, x_{q}, x_{q+1}, \ldots, x_{q+s}\right) \mid\right. \\
& x_{1}, \ldots, x_{q} \in \mathbb{R}^{n}, x_{q+1}, \ldots, x_{q+s} \in \mathbb{R}^{n+2}, \\
& x_{i} \neq x_{j} \quad \text { if } i, j \geq q+1, i \neq j, \\
& \psi\left(x_{i}\right) \neq \psi\left(x_{j}\right) \quad \text { if } i, j \leq q, i \neq j, \\
& \left.\psi\left(x_{i}\right) \neq x_{j} \quad \text { if } i \leq q, j \geq q+1\right\} .
\end{aligned}
$$

Then the principal faces corresponding to the contractions in (22) are identified with the following spaces.

\begin{tabular}{|c|c||c|c|}
\hline type & space & type & space \\
\hline$(1)$ & $S^{n+1} \times C_{\delta_{e} \Gamma}$ & $(5)$ & $S^{n+1} \times C_{\delta_{e} \Gamma}$ \\
$(2)$ & $S^{n-1} \times C_{\delta_{e} \Gamma}$ & $(6)$ & $S^{n-1} \times C_{\delta_{e} \Gamma}$ \\
$(3)$ & $S^{n+1} \times C_{\delta_{e} \Gamma}$ & $(7)$ & $S^{n-1} \times C_{\delta_{e} \Gamma}$ \\
$(4)$ & $S^{n-1} \times C_{\delta_{e} \Gamma}$ & & \\
\hline
\end{tabular}

Note that if $n$ is even, $C_{\delta_{e} \Gamma}$ can be canonically oriented. If $n$ is odd, we have the following lemma.

Lemma A.2 Suppose $n$ is odd. Let $\Gamma_{x}$ be a vertex oriented quasi Jacobi diagram with one exceptional vertex $x$ and $\bar{\Gamma}_{x}$ be $\Gamma_{x}$ with its vertex orientation at one external vertex reversed. Then there exists a choice of an orientation $\Omega=\Omega\left(\Gamma_{x}\right)$ on $C_{\Gamma_{x}}(\psi)$ such that

$$
\Omega\left(\bar{\Gamma}_{x}\right)=-\Omega\left(\Gamma_{x}\right)
$$

Proof If the $\theta$ part of $\Gamma_{x}$ is uni-trivalent except for one external tetravalent vertex $x$, where four edges $e_{1}, e_{2}, e_{3}, e_{4}$ with $\partial e_{i}=\left(v_{i}, x\right)$ for $i=1,2,3, \partial e_{4}=\left(x, v_{4}\right)$, $o_{x}\left(e_{i}\right)=i$ meet, set

$$
\begin{aligned}
\Omega_{x} & :=d X_{x}^{1} \wedge \ldots \wedge d X_{x}^{n+2} \wedge \Omega_{\bar{e}_{1-}} \wedge \Omega_{\bar{e}_{2-}} \wedge \Omega_{\bar{e}_{3-}} \wedge \Omega_{\bar{e}_{4+}}, \\
\Omega\left(\Gamma_{x}\right) & :=\Omega_{x} \wedge \bigwedge_{\substack{e \in E_{\theta}(\Gamma) \backslash \\
\left\{e_{1}, e_{2}, e_{3}, e_{4}\right\}}} \Omega_{e} .
\end{aligned}
$$

Algebraic $\mathcal{E} \mathcal{G}$ Geometric Topology, Volume 7 (2007) 
If the $\theta$ part of $\Gamma_{x}$ is uni-trivalent except for one internal bivalent vertex $x$, where two edges $e_{1}, e_{2}$ with $\partial e_{i}=\left(v_{i}, x\right), o_{x}\left(e_{i}\right)=i$ meet, set

$$
\begin{aligned}
\Omega_{x} & :=d X_{x}^{1} \wedge \ldots \wedge d X_{x}^{n} \wedge \Omega_{\bar{e}_{1-}} \wedge \Omega_{\bar{e}_{2-}}, \\
\Omega\left(\Gamma_{x}\right) & :=\Omega_{x} \wedge \bigwedge_{\substack{e \in E_{\theta}(\Gamma) \backslash \\
\left\{e_{1}, e_{2}\right\}}} \Omega_{e} .
\end{aligned}
$$

The cases of other edge orientations are similar.

If $\Gamma_{x}$ has an exceptional vertex $x$ having 2 or 3 incident $\eta$-edges, we define

$$
\begin{aligned}
\Omega_{x} & :=d X_{x}^{1} \wedge \ldots \wedge d X_{x}^{n}, \\
\Omega\left(\Gamma_{x}\right) & :=\Omega_{x} \wedge \bigwedge_{e \in E_{\theta}(\Gamma)} \Omega_{e} .
\end{aligned}
$$

Then the property (24) follows from the definition.

\section{A.4.3 Canceling of the integral along principal faces}

Lemma A.3 Suppose $n$ is odd. Let $\Gamma$ be a vertex oriented Jacobi diagram and $e$ be an edge of $\Gamma$. Then the orientation of the principal face induced from the orientation $\Omega(\Gamma)$ is as follows.

\begin{tabular}{|r|c|r||r|c|r|}
\hline type & space & induced orientation & type & space & induced orientation \\
\hline$(1)$ & $S^{n+1} \times C_{\delta_{e} \Gamma}$ & $\omega_{n+1} \wedge \Omega\left(\delta_{e} \Gamma\right)$ & $(5)$ & $S^{n+1} \times C_{\delta_{e} \Gamma}$ & $-\omega_{n+1} \wedge \Omega\left(\delta_{e} \Gamma\right)$ \\
$(2)$ & $S^{n-1} \times C_{\delta_{e} \Gamma}$ & $-\omega_{n-1} \wedge \Omega\left(\delta_{e} \Gamma\right)$ & $(6)$ & $S^{n-1} \times C_{\delta_{e} \Gamma}$ & $\omega_{n-1} \wedge \Omega\left(\delta_{e} \Gamma\right)$ \\
$(3)$ & $S^{n+1} \times C_{\delta_{e} \Gamma}$ & $\omega_{n+1} \wedge \Omega\left(\delta_{e} \Gamma\right)$ & $(7)$ & $S^{n-1} \times C_{\delta_{e} \Gamma}$ & $\omega_{n-1} \wedge \Omega\left(\delta_{e} \Gamma\right)$ \\
(4) & $S^{n-1} \times C_{\delta_{e} \Gamma}$ & $\omega_{n-1} \wedge \Omega\left(\delta_{e} \Gamma\right)$ & & & \\
\hline
\end{tabular}

Proof Let $\Gamma_{1}$ be as in the LHS of case (1) and we consider the orientation induced on the principal face $\mathcal{S}_{\Gamma_{1}, e_{1}}$ corresponding to the contraction of the edge $e_{1}=(w, y)$. The orientation of $C_{\Gamma_{1}}$ is

$$
\begin{aligned}
\Omega\left(\Gamma_{1}\right) & =\left(d X_{y}^{1} d^{n} X_{w}\right) \wedge\left(d X_{y}^{2} \Omega_{\bar{e}_{v}}\right) \wedge\left(\Omega_{\bar{e}_{u}} d X_{y}^{3} \ldots d X_{y}^{n+2}\right) \wedge(\text { the rest }) \\
& =d X_{y}^{1} \ldots d X_{y}^{n+2} \wedge d^{n} X_{w} \Omega_{\bar{e}_{v}} \Omega_{\bar{e}_{u}} \wedge(\text { the rest }) .
\end{aligned}
$$

Let $n_{1}$ be the outgoing unit normal vector field on $S^{n+1}$. Then the ingoing normal vector field on the principal face is given by $n_{1}$. The induced orientation of $\mathcal{S}_{\Gamma_{1}, e_{1}}$ is then

$$
i_{n_{1}} \Omega\left(\Gamma_{1}\right)=\omega_{n+1}(\alpha) \wedge d^{n} X_{w} \Omega_{\bar{e}_{v}} \Omega_{\bar{e}_{u}} \wedge(\text { the rest })=\omega_{n+1}(\alpha) \wedge \Omega\left(\delta_{e_{1}} \Gamma_{1}\right)
$$


where $\alpha=\frac{X_{y}-X_{w}}{\left|X_{y}-X_{w}\right|}$. For cases (3) and (5), the results follow from the following:

$$
\begin{aligned}
& i_{n_{1}} \Omega\left(\Gamma_{3}\right)=\omega_{n+1} d^{n} X_{w} \Omega_{\bar{e}_{v}} \Omega_{\bar{e}_{u}} \wedge(\text { the rest }) \\
& i_{n_{1}} \Omega\left(\Gamma_{5}\right)=-\omega_{n+1} d^{n+2} X_{x} \Omega_{\bar{e}_{b}} \Omega_{\bar{e}_{a}} \Omega_{\bar{e}_{c}} \Omega_{\bar{e}_{d}} \wedge \text { (the rest) }
\end{aligned}
$$

Let $\Gamma_{2}$ be as in the LHS of case (2) and we consider the orientation induced on the principal face $\mathcal{S}_{\Gamma_{2}, e_{2}}$ corresponding to the contraction of the edge $e_{2}=(w, j)$. The orientation of $C_{\Gamma_{2}}$ is

$$
\Omega\left(\Gamma_{2}\right)=d^{n} X_{w} \Omega_{\bar{e}_{v}} \Omega_{\bar{e}_{u}} d^{n} X_{j} \wedge \text { (the rest) }=d^{n} X_{w} d^{n} X_{j} \Omega_{\bar{e}_{v}} \Omega_{\bar{e}_{u}} \wedge \text { (the rest). }
$$

Let $n_{2}$ be the outgoing unit normal vector field on $S^{n-1}$. Then the induced orientation of $\mathcal{S}_{\Gamma_{2}, e_{2}}$ is

$$
i_{n_{2}} \Omega\left(\Gamma_{2}\right)=-\omega_{n-1}(\beta) \wedge d^{n} X_{w} \Omega_{\bar{e}_{v}} \Omega_{\bar{e}_{u}} \wedge(\text { the rest })=-\omega_{n-1}(\beta) \wedge \Omega\left(\delta_{e_{2}} \Gamma_{2}\right)
$$

where $\beta=\frac{X_{j}-X_{w}}{\left|X_{j}-X_{w}\right|}$. For case (4) the result follows similarly from the following:

$$
i_{n_{2}} \Omega\left(\Gamma_{4}\right)=\omega_{n-1} \Omega_{\bar{e}_{w}} \Omega_{\bar{e}_{v}} \Omega_{\bar{e}_{u}} \wedge(\text { the rest })
$$

Let $\Gamma_{6}$ be as in the LHS of case (6) and we consider the orientation induced on the principal face $\mathcal{S}_{\Gamma_{6}, e_{6}}$ corresponding to the contraction of the edge $e_{6}=(i, j)$. The orientation of $C_{\Gamma_{6}}$ is

$$
\Omega\left(\Gamma_{6}\right)=d^{n} X_{j} d^{n} X_{i} \wedge \text { (the rest). }
$$

The induced orientation of $\mathcal{S}_{\Gamma_{6}, e_{6}}$ is

$$
i_{n_{2}} \Omega\left(\Gamma_{6}\right)=\omega_{n-1}(\gamma) d^{n} X_{i} \wedge(\text { the rest })=\omega_{n-1}(\gamma) \wedge \Omega\left(\delta_{e_{6}} \Gamma_{6}\right)
$$

where $\gamma=\frac{X_{j}-X_{i}}{\left|X_{j}-X_{i}\right|}$. Case (7) is similar.

Lemma A.4 Let $\left(\Gamma_{i}, e_{i}\right)$, for $i=1, \ldots, 7$, be the pair of the Jacobi diagram and the edge to be contracted on it, as in the LHS of case (i) in (22). Then we have

$$
I\left(\Gamma_{1}, e_{1}\right)=-I\left(\Gamma_{2}, e_{2}\right), \quad I\left(\Gamma_{3}, e_{3}\right)=-I\left(\Gamma_{4}, e_{4}\right), \quad I\left(\Gamma_{6}, e_{6}\right)=I\left(\Gamma_{7}, e_{7}\right) .
$$

Proof By definition,

$$
\begin{aligned}
I\left(\Gamma_{1}, e_{1}\right) & =\int_{S^{n+1} \times C_{\delta_{1}} \Gamma_{1}} \theta_{e_{1}} \wedge \lambda_{2}\left(\delta_{e_{1}} \Gamma_{1}\right) \\
& =\int_{S^{n+1}} \theta_{e_{1}} \int_{C_{\delta_{e_{1}} \Gamma_{1}}} \lambda_{2}\left(\delta_{e_{1}} \Gamma_{1}\right)=\int_{C_{\delta_{e_{1}} \Gamma_{1}}} \lambda_{2}\left(\delta_{e_{1}} \Gamma_{1}\right)
\end{aligned}
$$

Algebraic $8 \mathcal{G}$ Geometric Topology, Volume 7 (2007) 
where $\theta_{e_{1}}$ denotes the pullback form of $\omega_{n+1}$ via the Gauss map $u$ with respect to $e_{1}$. Similarly we have

$$
I\left(\Gamma_{2}, e_{2}\right)=\int_{C_{\delta_{e_{2}} \Gamma_{2}}} \lambda_{2}\left(\delta_{e_{2}} \Gamma_{2}\right) .
$$

When $n$ is even, the induced orientations on $C_{\delta_{e_{1}} \Gamma_{1}} \cong C_{\delta_{e_{2}} \Gamma_{2}}$ are the same, and when $n$ is odd, the induced orientations on $C_{\delta_{e_{1}} \Gamma_{1}} \cong C_{\delta_{e_{2}} \Gamma_{2}}$ are opposite by Lemma A.3. So it suffices to prove $\lambda_{2}\left(\delta_{e_{1}} \Gamma_{1}\right)=(-1)^{n-1} \lambda_{2}\left(\delta_{e_{2}} \Gamma_{2}\right)$. In terms of the labels of vertices in (22), we have

$$
\omega\left(\Gamma_{1}\right)=\theta_{w y} \wedge \theta_{v y} \wedge \omega((y, u)) \wedge(\text { the rest }) .
$$

On the principal face corresponding to the contraction of $e_{1}=(w, y)$, this form becomes $\theta_{e_{1}} \wedge \theta_{v w} \omega((w, u)) \wedge($ the rest $)$ and thus $\lambda_{2}\left(\delta_{e_{1}} \Gamma_{1}\right)=\theta_{v w} \omega((w, u)) \wedge($ the rest $)$. On the other hand, we have

$$
\omega\left(\Gamma_{2}\right)=\theta_{v w} \eta_{w j} \omega((j, u))=(-1)^{n-1} \eta_{w j} \theta_{v w} \omega((j, u)) \wedge(\text { the rest }) .
$$

Thus $\lambda_{2}\left(\delta_{e_{2}} \Gamma_{2}\right)=(-1)^{n-1} \theta_{v w} \omega((w, u)) \wedge($ the rest $) \equiv(-1)^{n-1} \lambda_{2}\left(\delta_{e_{1}} \Gamma_{1}\right)$.

The second and the third identity may be similarly proved from the facts:

(1) induced orientations on $C_{\delta_{e_{3}} \Gamma_{3}} \cong C_{\delta_{e_{4}} \Gamma_{4}}$ are the same,

(2) $\lambda_{2}\left(\delta_{e_{3}} \Gamma_{3}\right)=(-1)^{n} \omega_{n+1} \theta_{v w} \theta_{u w}$ (rest),

(3) $\lambda_{2}\left(\delta_{e_{4}} \Gamma_{4}\right)=(-1)^{n-1} \omega_{n-1} \theta_{v w} \theta_{u w}$ (rest),

(4) induced orientations on $C_{\delta_{e_{6}} \Gamma_{7}} \cong C_{\delta_{e_{6}} \Gamma_{7}}$ are the same,

(5) $\lambda_{2}\left(\delta_{e_{6}} \Gamma_{6}\right)=\lambda_{2}\left(\delta_{e_{7}} \Gamma_{7}\right)$

Proof of Proposition A.1 First we show that if the two vertices in $A$ are not connected by an edge, then $I(\Gamma, A)=0$. By (16) or (17), $\mathcal{S}_{\Gamma, A}$ is a sphere bundle over $C_{\Gamma / A}$ and from Section A.3, $\omega(\Gamma)$ is basic in this bundle, namely a pullback of some form on $C_{\Gamma / A}$ with less dimension. Hence $I(\Gamma, A)=0$.

Then it is enough to prove for the faces corresponding to the collapse of an edge of $\Gamma$. The proof is divided according to the cases in the definition of $\delta_{e}$.

Cases (1) and (2) (Appearance of the ST relation) For cases (1) and (2), we will see that the contributions of the faces corresponding to the following pair of contractions of graphs cancel each other:

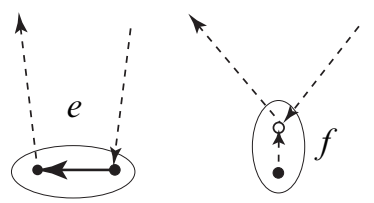

Algebraic $8 \mathcal{G}$ Geometric Topology, Volume 7 (2007) 
which we denote by $\left(\Gamma^{\mathrm{a}}, e\right),\left(\Gamma^{\mathrm{b}}, f\right)$ respectively.

From (23), the face of $\left(\Gamma^{\mathrm{a}}, e\right)$ is identified with $S^{n-1} \times C_{\delta_{e} \Gamma^{\mathrm{a}}}$, where the factor $S^{n-1}$ is the relative configuration space of 2 points on $T_{x} \mathbb{R}^{n}$. The face of $\left(\Gamma^{\mathrm{b}}, f\right)$ is identified with $S^{n+1} \times C_{\delta_{f} \Gamma^{\mathrm{b}}}$, where the factor $S^{n+1}$ is the relative configuration space of 2 points on $\mathbb{R}^{n+2}$.

Let $n_{\mathrm{a}}$ be the number of $\eta$-edges $e^{\prime}$ on $\Gamma^{\mathrm{a}}$ such that $\delta_{e} \Gamma^{\mathrm{a}}=\delta_{e^{\prime}} \Gamma^{\mathrm{a}} . n_{\mathrm{b}}$ is similarly defined to be the number of $\eta$-edges $f^{\prime}$ on $\Gamma^{\mathrm{b}}$ such that $\delta_{f} \Gamma^{\mathrm{b}}=\delta_{f^{\prime}} \Gamma^{\mathrm{b}}$. Then we have

$$
\frac{\mid \text { Aut } \Gamma^{\mathrm{a}} \mid}{n_{\mathrm{a}}}=\frac{\mid \text { Aut } \Gamma^{\mathrm{b}} \mid}{n_{\mathrm{b}}}=\mid \text { Aut } \delta_{e} \Gamma^{\mathrm{a}}|=| \text { Aut } \delta_{f} \Gamma^{\mathrm{b}} \mid \text {. }
$$

We assume that each automorphism in Aut $\Gamma^{\mathrm{a}}$ preserves the orientation of $C_{\Gamma^{\mathrm{a}}}$ since if not $I\left(\Gamma^{\mathrm{a}}, e\right)=0$. The case of $\Gamma^{\mathrm{b}}$ is similar. Since the integration along the sphere factors contributes by $1, z_{k}$ restricted to these faces is

$$
\frac{n_{\mathrm{a}} I\left(\Gamma^{\mathrm{a}}, e\right) w_{k}\left(\Gamma^{\mathrm{a}}\right)}{\mid \text { Aut } \Gamma^{\mathrm{a}} \mid}+\frac{n_{\mathrm{b}} I\left(\Gamma^{\mathrm{b}}, f\right) w_{k}\left(\Gamma^{\mathrm{b}}\right)}{\mid \text { Aut } \Gamma^{\mathrm{b}} \mid}=\frac{I\left(\Gamma^{\mathrm{a}}, e\right)}{\left|\operatorname{Aut} \delta_{e} \Gamma^{\mathrm{a}}\right|}\left(w_{k}\left(\Gamma^{\mathrm{a}}\right)-w_{k}\left(\Gamma^{\mathrm{b}}\right)\right)=0,
$$

where $I\left(\Gamma^{\mathrm{b}}, f\right)=-I\left(\Gamma^{\mathrm{a}}, e\right)$ by Lemma A.4 and by the ST relation.

Cases (3) and (4) (Appearance of the ST2 relation) For cases (3) and (4), we will see that the contributions of the faces corresponding to the following pair of graphs cancel each other:

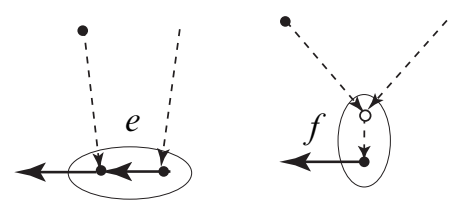

which we denote by $\left(\Gamma^{\mathrm{c}}, e\right),\left(\Gamma^{\mathrm{d}}, f\right)$ respectively. From (23), the faces corresponding to $\left(\Gamma^{\mathrm{c}}, e\right),\left(\Gamma^{\mathrm{d}}, f\right)$ can be identified with the spaces

$$
\begin{gathered}
\left(\Gamma^{\mathrm{c}}, e\right): S^{n-1} \times C_{\delta_{e} \Gamma^{\mathrm{c}}}, \\
\left(\Gamma^{\mathrm{d}}, f\right): S^{n+1} \times C_{\delta_{f} \Gamma^{\mathrm{d}}} .
\end{gathered}
$$

Let $n_{\mathrm{c}}$ be the number of $\eta$-edges $e^{\prime}$ on $\Gamma^{\mathrm{c}}$ such that $\delta_{e} \Gamma^{\mathrm{c}}=\delta_{e^{\prime}} \Gamma^{\mathrm{c}} . n_{\mathrm{d}}$ is similarly defined. Then we have

$$
\frac{\mid \text { Aut } \Gamma^{\mathrm{c}} \mid}{n_{\mathrm{c}}}=\frac{\mid \text { Aut } \Gamma^{\mathrm{d}} \mid}{n_{\mathrm{d}}}=\mid \text { Aut } \delta_{e} \Gamma^{\mathrm{c}}|=| \text { Aut } \delta_{f} \Gamma^{\mathrm{d}} \mid .
$$

We assume that each automorphism in Aut $\Gamma^{\mathrm{c}}$ preserves the orientation of $C_{\Gamma^{\mathrm{c}}}$ since if not $I\left(\Gamma^{\mathrm{c}}, e\right)=0$. The case of $\Gamma^{\mathrm{d}}$ is similar. Note that some of them may not be 


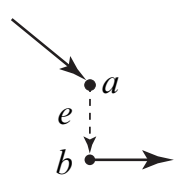

(a)

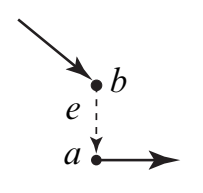

(a’)

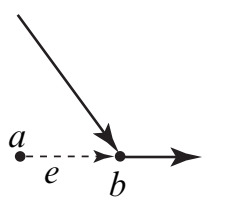

(b)

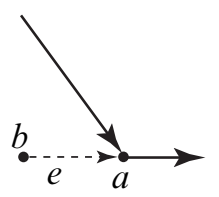

(b')

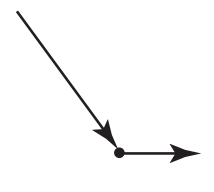

(c)

Figure 7: Possible graphs contracted into (c)

Jacobi diagrams. Since the integration along the sphere factors contributes by $1, z_{k}$ restricted to these faces is

$$
\frac{n_{\mathrm{c}} I\left(\Gamma^{\mathrm{c}}, e\right) w_{k}\left(\Gamma^{\mathrm{c}}\right)}{\mid \text { Aut } \Gamma^{\mathrm{c}} \mid}+\frac{n_{\mathrm{d}} I\left(\Gamma^{\mathrm{d}}, f\right) w_{k}\left(\Gamma^{\mathrm{d}}\right)}{\left|\operatorname{Aut} \Gamma^{\mathrm{d}}\right|}=\frac{I\left(\Gamma^{\mathrm{c}}, e\right)}{\left|\operatorname{Aut} \delta_{e} \Gamma^{\mathrm{c}}\right|}\left(w_{k}\left(\Gamma^{\mathrm{c}}\right)-w_{k}\left(\Gamma^{\mathrm{d}}\right)\right)=0,
$$

where $I\left(\Gamma^{\mathrm{d}}, f\right)=-I\left(\Gamma^{\mathrm{c}}, e\right)$ by Lemma A.4, and by the ST2 relation.

Cases (6) and (7) (Appearance of the C relation) Then $z_{k}$ can be written without using $\mid$ Aut $\Gamma \mid$ as

$$
z_{k}=\frac{1}{(2 k) !} \sum_{\Gamma: \text { labeled }} I(\Gamma) w_{k}(\Gamma),
$$

since there are $(2 k) ! / \mid$ Aut $\Gamma \mid$ ways of labeling the $2 k$ vertices with distinguished labels in $\{1,2, \ldots, 2 k\}$. There are possibly 4 labeled graphs $\Gamma_{1}^{\mathrm{L}}, \Gamma_{2}^{\mathrm{L}}, \Gamma_{1}^{\mathrm{R}}, \Gamma_{2}^{\mathrm{R}}$ as in Figure $7(\mathrm{a}),(\mathrm{a}),(\mathrm{b})$ and (b') respectively, which are mapped by $\delta_{e}$ to the graph as in Figure 7(c). From (23), the corresponding principal faces can be identified with the following spaces via the tangent framing:

$$
S^{n-1} \times C_{\delta_{e} \Gamma_{1}^{\mathrm{L}}}, \quad S^{n-1} \times C_{\delta_{e} \Gamma_{2}^{\mathrm{L}}}, \quad S^{n-1} \times C_{\delta_{e} \Gamma_{1}^{\mathrm{R}},}, \quad S^{n-1} \times C_{\delta_{e} \Gamma_{2}^{\mathrm{R}}}
$$

Since the integration along $S^{n-1}$ fiber contributes by some common 2 -form $\mu$ on $I_{n}\left(\mathbb{R}^{n+2}\right)$, the integrals restricted to these faces are

$$
\begin{aligned}
& \frac{1}{(2 k) !}\left(I\left(\Gamma_{1}^{\mathrm{L}}, e\right) w_{k}\left(\Gamma_{1}^{\mathrm{L}}\right)+I\left(\Gamma_{2}^{\mathrm{L}}, e\right) w_{k}\left(\Gamma_{2}^{\mathrm{L}}\right)+I\left(\Gamma_{1}^{\mathrm{R}}, e\right) w_{k}\left(\Gamma_{1}^{\mathrm{R}}\right)+I\left(\Gamma_{2}^{\mathrm{R}}, e\right) w_{k}\left(\Gamma_{2}^{\mathrm{R}}\right)\right) \\
& \quad=\frac{1}{(2 k) !}\left(w_{k}\left(\Gamma_{1}^{\mathrm{L}}\right)+w_{k}\left(\Gamma_{2}^{\mathrm{L}}\right)+w_{k}\left(\Gamma_{1}^{\mathrm{R}}\right)+w_{k}\left(\Gamma_{2}^{\mathrm{R}}\right)\right) \int_{C_{\delta_{e} \Gamma_{1}^{\mathrm{L}}}} \varphi^{*} \mu \wedge \lambda_{2}\left(\delta_{e} \Gamma_{1}^{\mathrm{L}}\right)=0,
\end{aligned}
$$

where $I\left(\Gamma_{i}^{\mathrm{L}}, e\right)=I\left(\Gamma_{i}^{\mathrm{R}}, e\right)$ by Lemma A.4 and by the $\mathrm{C}$ relation.

Case (5) There are just 6 labeled graphs $\Gamma_{1}^{\mathrm{I}}, \Gamma_{2}^{\mathrm{I}}, \Gamma_{1}^{\mathrm{H}}, \Gamma_{2}^{\mathrm{H}}, \Gamma_{1}^{\mathrm{X}}, \Gamma_{2}^{\mathrm{X}}$ as in Figure 8(a), (a'), (b), (b'), (c) and (c') respectively, which are mapped by $\delta_{e}$ to the graph $\Gamma^{\mathrm{d}}$ as in Figure 8(d) (the - signs of (b) and (b') denote the reversion of the vertex orientation at one vertex, and are because the cyclic permutation of 4 edges is an odd permutation). 


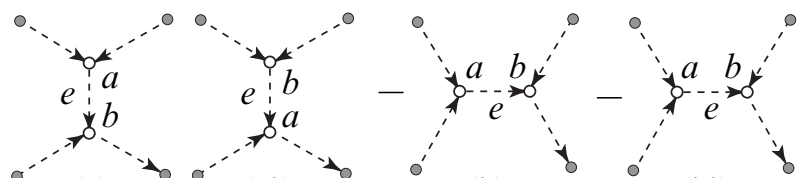

(a)

(a')

(b)

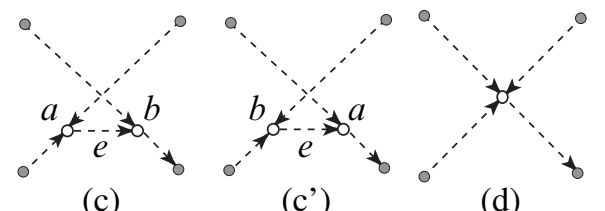

(c)

$\left(c^{\prime}\right)$

(d)

Figure 8: Possible graphs contracted into (d)

Since two of the three edges incident to the tetra-valent vertex of $\Gamma^{\mathrm{d}}$ are always hairs on a subgraph as in (2)(b), the involution exchanging the two hairs preserves the integrand form and reverses the orientation of the configuration space if $n$ is odd, or reverses the sign of the integrand form and preserves the orientation of the configuration space if $n$ is even. Hence the integral over such faces are self-cancelling.

\section{A.5 Hidden faces}

Now we briefly review the proof of the vanishing of $I(\Gamma) w_{k}(\Gamma)$ restricted to the hidden faces, where the proof is explicitly given by Rossi [15]. Let $A \subset V(\Gamma),|A| \geq 3$ be a set of points to be collapsed having external $\theta$-edges ( $\theta$-edges connecting points in $A$ and points not in $A$ ) and let $\Gamma_{A}$ be the subgraph of $\Gamma$ consisting of edges connecting two vertices in $A$. The goal of this subsection is to prove the following proposition.

Proposition A.5 The term $I(\Gamma) w_{k}(\Gamma)$ restricted to any face corresponding to the diagonal where the points in $A \subset V(\Gamma)$ with $A \neq V(\Gamma),|A| \geq 3$ coincide, vanishes.

The proof of Proposition A.5 is done by using the Vanishing Lemmata A.8, A.9 and A.10 below, proved in [15] and by Cattaneo, Cotta-Ramusino and Longoni [6].

Lemma A.6 If $|A| \geq 3$ and $A$ has external $\theta$-edges, then the integral restricted to the corresponding face vanishes.

Proof By Lemma A.7 below, we need only to consider the cases in which $\Gamma_{A}$ is connected. 
Let $a$ be a vertex in $\Gamma_{A}$ which is an end of an external $\theta$-edge. The possible cases in which $\Gamma_{A}$ are connected are as in the following picture:

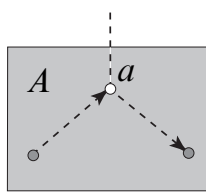

(1)

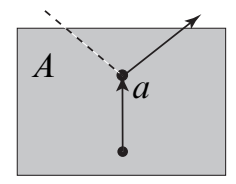

(5)

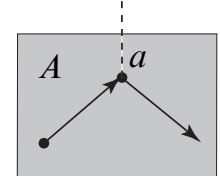

(2)

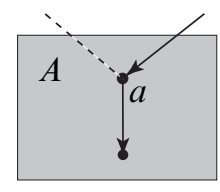

(6)

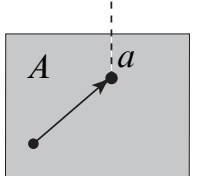

(3)

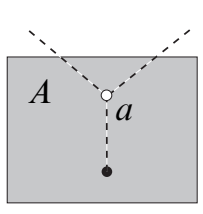

(7)

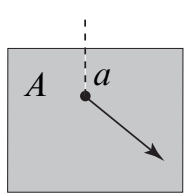

(4)

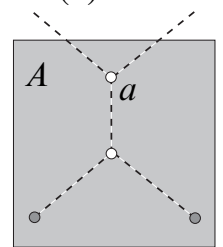

(8)

The reasons for the vanishing of the integral $I(\Gamma)$ along $\mathcal{S}_{\Gamma, A}$ are as follows.

Cases (1) and (2) By Lemma A.8 below.

Cases (3)-(8) By Lemma A.9 below.

Lemma A.7 If $\Gamma_{A}$ is not connected, then the integral restricted to $\mathcal{S}_{\Gamma, A}$ vanishes.

Proof By the observations in Section A.1, the face $\mathcal{S}_{\Gamma, A}$ is a bundle $\mathcal{S}_{\Gamma_{A}} \rightarrow C_{\Gamma / A}$ with fiber $F_{A}$ the configuration space associated to the subgraph $\Gamma_{A}$ modulo translations and dilations. Since $\Gamma_{A}$ is not connected, the action $T$, acting on $F_{A}$ as translations of one connected component in $\Gamma_{A}$ fixing all other components, is defined. The condition $|A| \geq 3$ implies that this action is non trivial on $F_{A}$ (hence independent of the overall translations acting on $F_{A}$ ) and the quotient map $F_{A} \rightarrow F_{A} / T$ gives a bundle $\pi^{T}: \mathcal{S}_{\Gamma_{A}} \rightarrow \mathcal{S}_{\Gamma_{A}} / T$ with $n$ or $(n+2)$ dimensional fiber, depending on whether the translated component has internal vertices or not. Since the $T$-action does not affect the integrand form $\omega(\Gamma)$ extended to $\mathcal{S}_{\Gamma, A}$, namely the integrand form can be written as $\left(\pi^{T}\right)^{*} \omega(\Gamma)^{T}$ for some form $\omega(\Gamma)^{T}$ on $\mathcal{S}_{\Gamma, A} / T$. Thus

$$
\int_{\mathcal{S}_{\Gamma, A}} \omega(\Gamma)=\int_{\mathcal{S}_{\Gamma}}\left(\pi^{T}\right)^{*} \omega(\Gamma)^{T}=\int_{\mathcal{S}_{\Gamma} / T} \omega(\Gamma)^{T}
$$

where the integration is the fiber integration. The last integral is over the space with codimension at least $(n+1)$ and so it vanishes.

Proof of Proposition A.5 By Lemma A.6 and Proposition 3.1, for a non vanishing face the $\theta$ part of $\Gamma_{A}$ must form a disjoint union of chords, trees and wheels entirely included in $\Gamma_{A}$. So we assume this condition in the rest of the proof. 


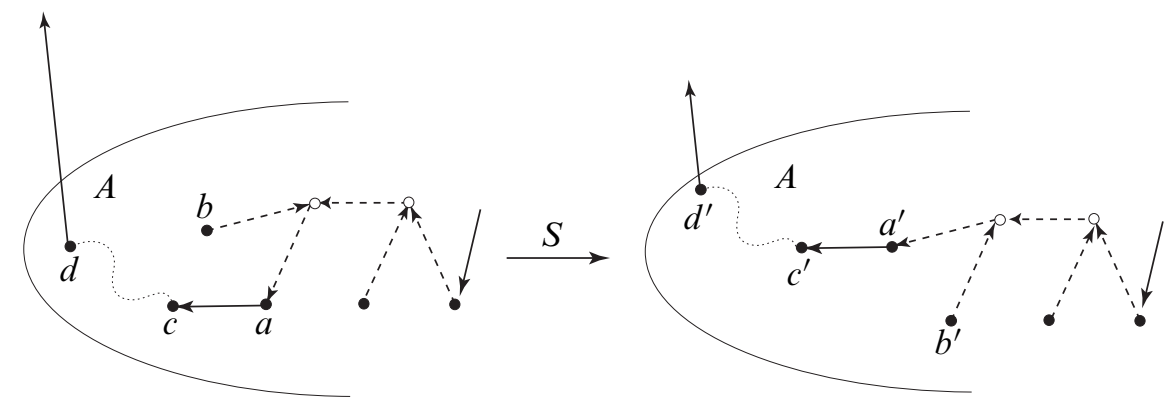

Figure 9: $\left(x_{d}, \ldots, x_{c}, x_{a}, x_{b}, \ldots\right) \mapsto\left(x_{d}+\left(x_{b}-x_{a}\right), \ldots, x_{c}+\left(x_{b}-x_{a}\right), x_{b}, x_{a}, \ldots\right)$

We may assume that there exists an $\eta$-edge going from a point in $A$ to a point not in $A$. Then $\Gamma_{A}$ consists of a sequence of subgraphs as in (2)(b) joined by $\eta$-edges. If $\Gamma_{A}$ has a tree component with at least one external vertex, then we define the following involution on $\widehat{B}_{A}$ as in Figure 9: let us write the points in the relative configuration as $\left(x_{d}, \ldots, x_{c}, x_{a}, x_{b}, \ldots\right)$, where the ' $\ldots$ ' between $x_{d}$ and $x_{c}$ are all points which belong to the snake-like piece $c \sim d$ in the graph, and all other '.. ' denote other points. Then the involution takes the form

$$
\begin{aligned}
x_{e} & \mapsto x_{e}+\left(x_{b}-x_{a}\right) \quad \text { if } e \text { is a vertex on the piece } c \sim d \text { of the graph, } \\
\text { or } x_{a} & \mapsto x_{b}, \\
x_{b} & \mapsto x_{a}, \\
\text { and } x_{f} & \mapsto x_{f} \quad \text { otherwise. }
\end{aligned}
$$

This involution reverses (resp. preserves) the orientation of the fiber of $\widehat{B}_{A}$ and preserves (resp. reverses) the sign of the form if $n$ is odd (resp. even). (Here, the path $c \sim d$ in Figure 9 is disjoint from the depicted tree component and from the invisible part, by the definition of Jacobi diagrams and by the above assumptions, and $b$ may be assumed to be univalent.) Hence the integral vanishes. If $\Gamma$ has only chords in its $\theta$ part, then by the assumption, there has to be at least two chords connected by an $\eta$-edge. Hence there must be a subgraph of one of Lemma A.10.

The following lemma is an analogue of a lemma of Kontsevich [13], which is proved by Rossi [15]. 
Lemma A.8 If $\Gamma_{A}$ has a subgraph of one of the following forms:
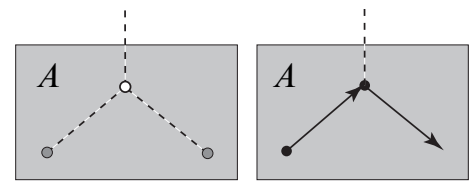

where the gray vertices may both be internal and external, then the integral restricted to $\mathcal{S}_{\Gamma, A}$ vanishes.

Lemma A.8 is proved by using the central symmetry with center the middle of the two gray vertices acting on the white vertex in the first case, and a similar one in the second case. By these symmetries, the integral restricted to $\mathcal{S}_{\Gamma, A}$ is self-cancelling.

The following two lemmas are proved explicitly in [15].

Lemma A.9 If $|A| \geq 3$ and $\Gamma_{A}$ has a subgraph of one of the following forms:
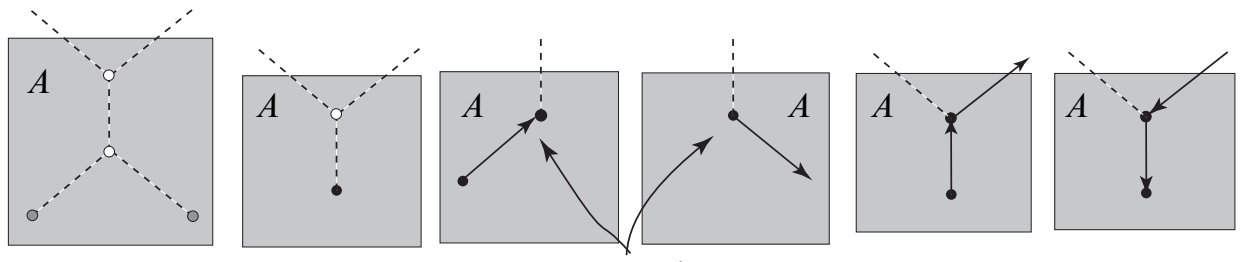

no more edges

then the integral restricted to $\mathcal{S}_{\Gamma, A}$ vanishes.

Lemma A.9 is proved by showing that the quotient space of $\mathcal{S}_{\Gamma, A}$ by the action rescaling the central edge in the box, which does not affect the integrand form, has strictly less dimension than $\mathcal{S}_{\Gamma, A}$.

Lemma A.10 If $\Gamma_{A}$ has a subgraph of one of the forms
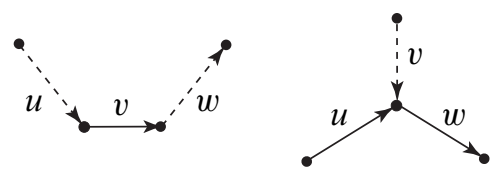

then the integral restricted to $\mathcal{S}_{\Gamma, A}$ vanishes.

Lemma A.10 is proved by using the symmetry as in Lemma A.8, carrying the vectors $(u, v, w)$ to $(w, v, u)$, cancelling the integral with itself. More precisely, the symmetry for the first case is given as a composition of the reversion of the two boundary vertices 
of $v$ and the involution in Lemma A.8 for the two vertices with respect to the remaining vertices. The symmetry for the second case is given as a composition of the central symmetry for the vector $v$ with respect to the target of $v$, and the involution in Lemma A.8 for the two boundary vertices of the image of $v$.

\section{A.6 Infinite and anomalous faces}

For the infinite faces, the following proposition is proved in [15].

Proposition A.11 The integral $I(\Gamma)$ restricted to the infinite faces $\mathcal{S}_{\Gamma, \infty, A}$ vanishes.

This is proved by checking that the infinite faces have codimension at least 2 .

A.6.1 Anomaly correction in the case $n=2$ In the case $n=2$ and $k=3$, the following proposition is proved in $[7 ; 15]$, which is rather difficult part in the proof of the invariance, involving the analysis on invariant forms on the Stiefel and the Grassmann manifolds.

Proposition A.12 If $n=2$, then there exists a 2-form $\hat{\rho}(\Gamma)$ on $I_{2}\left(\mathbb{R}^{4}\right)$ such that

$$
d \int_{C_{1}} \varphi^{*} \hat{\rho}(\Gamma)=-I(\Gamma, V(\Gamma)) .
$$

Hence the addition of the correction term to $z_{3}$ :

$$
\widehat{z}_{3}(\psi):=z_{3}(\psi)+\sum_{\Gamma \in \mathcal{G}_{3}^{0}} \frac{\widetilde{\Theta}(\Gamma)(\psi) w_{3}(\Gamma)}{|\operatorname{Aut} \Gamma|}
$$

where $\widetilde{\Theta}(\Gamma)=\int_{C_{1}} \varphi^{*} \hat{\rho}(\Gamma)$, is proved to be $d$-closed.

A.6.2 Anomaly vanishing in the case $n$ odd We show the following proposition as already announced in [15]. Recall that

$$
I(\Gamma, V(\Gamma))=\int_{C_{1}} \varphi^{*} \pi_{*}^{\partial} \lambda_{1}(\Gamma) .
$$

Proposition A.13 $\pi_{*}^{\partial} \lambda_{1}(\Gamma)$ vanishes for any $\Gamma$ in the case $n$ is odd. 
Proof Note first that each $\Gamma$ has even number of vertices and even number of edges. Let $x_{1}, \ldots, x_{j}$ be internal vertices of $\Gamma$ mapped into an underlying $n$ dimensional plane with a tangent frame $f \in I_{n}\left(\mathbb{R}^{n+2}\right)$ and $y_{1}, \ldots, y_{k}$ be external vertices of $\Gamma$ in the ambient space $\mathbb{R}^{n+2}$ so that $\left(f ; x_{1}, \ldots, x_{j}, y_{1}, \ldots, y_{k}\right) \in \widehat{B}_{V(\Gamma)}$. Then consider the involution

$S:\left(f ; x_{1}, x_{2}, \ldots, x_{j}, y_{1}, \ldots, y_{k}\right)$

$$
\mapsto\left(f ; x_{1}, 2 x_{1}-x_{2}, \ldots, 2 x_{1}-x_{j}, 2 f\left(x_{1}\right)-y_{1}, \ldots, 2 f\left(x_{1}\right)-y_{k}\right)
$$

on the fiber. Since $j+k-1$ is odd if $n$ is odd, $S$ reverses the orientation of the fiber

of $\widehat{B}_{V(\Gamma)}$ and preserves the form $\lambda_{1}(\Gamma)$. Therefore the integration along the fiber vanishes as in the proof of Lemma A.8.

\section{References}

[1] D Altschuler, L Freidel, Vassiliev knot invariants and Chern-Simons perturbation theory to all orders, Comm. Math. Phys. 187 (1997) 261-287 MR1463829

[2] S Axelrod, I M Singer, Chern-Simons perturbation theory, from: "Proceedings of the XXth International Conference on Differential Geometric Methods in Theoretical Physics, Vol. 1, 2 (New York, 1991)", World Sci. Publ., River Edge, NJ (1992) 3-45 MR1225107

[3] D Bar-Natan, Perturbative aspects of the Chern-Simons field theory, $\mathrm{PhD}$ thesis, Princeton University (1991)

[4] R Bott, Configuration spaces and imbedding invariants, Turkish J. Math. 20 (1996) 1-17 MR1392659

[5] R Bott, C Taubes, On the self-linking of knots, J. Math. Phys. 35 (1994) 5247-5287 MR1295465Topology and physics

[6] A S Cattaneo, P Cotta-Ramusino, R Longoni, Configuration spaces and Vassiliev classes in any dimension, Algebr. Geom. Topol. 2 (2002) 949-1000 MR1936977

[7] A S Cattaneo, C A Rossi, Wilson surfaces and higher dimensional knot invariants, Comm. Math. Phys. 256 (2005) 513-537 MR2161270

[8] W Fulton, R MacPherson, A compactification of configuration spaces, Ann. of Math. (2) 139 (1994) 183-225 MR1259368

[9] E Guadagnini, M Martellini, M Mintchev, Wilson lines in Chern-Simons theory and link invariants, Nuclear Phys. B 330 (1990) 575-607 MR1043394

[10] K Habiro, T Kanenobu, A Shima, Finite type invariants of ribbon 2-knots, from: "Low-dimensional topology (Funchal, 1998)", Contemp. Math. 233, Amer. Math. Soc., Providence, RI (1999) 187-196 MR1701683 
[11] K Habiro, A Shima, Finite type invariants of ribbon 2-knots II, Topology Appl. 111 (2001) 265-287 MR1814229

[12] T Kohno, Vassiliev invariants and de Rham complex on the space of knots, from: "Symplectic geometry and quantization (Sanda and Yokohama, 1993)", Contemp. Math. 179, Amer. Math. Soc., Providence, RI (1994) 123-138 MR1319605

[13] M Kontsevich, Feynman diagrams and low-dimensional topology, from: "First European Congress of Mathematics, Vol. II (Paris, 1992)”, Progr. Math. 120, Birkhäuser, Basel (1994) 97-121 MR1341841

[14] S Poirier, The configuration space integral for links in $\mathbb{R}^{3}$, Algebr. Geom. Topol. 2 (2002) 1001-1050 MR1936978

[15] C Rossi, Invariants of higher-dimensional knots and topological quantum field theories, $\mathrm{PhD}$ thesis, Zurich University (2002)

[16] D Thurston, Integral expressions for the Vassiliev knot invariants, AB thesis, Harvard University (1995) arXiv:math.QA/9901110

[17] T Watanabe, Clasper-moves among ribbon 2-knots characterizing their finite type invariants, J. Knot Theory Ramifications 15 (2006) 1163-1199

[18] E Witten, Quantum field theory and the Jones polynomial, Comm. Math. Phys. 121 (1989) 351-399 MR990772

Research Institute for Mathematical Sciences, Kyoto University

Kyoto, Japan

tadayuki@kurims.kyoto-u.ac.jp

Received: 3 February 2006 Revised: 8 June 2006 